\title{
Filtro Híbrido Adaptativo en Cascada para Arreglos Lineales Uniformes de Antenas
}

\author{
Walter Orozco-Tupacyupanqui, Mariko Nakano-Miyatake y Héctor Pérez-Meana* \\ Escuela Superior de Ingeniería Mecánica y Eléctrica, Instituto Politécnico Nacional, Av. Santa Ana 1000, \\ Col. San Francisco Culhuacán, 04430 México D.F., México. \\ (waltozco@yahoo.com,mnakano@ipn.mx,hmperezm@ipn.mx ) \\ ${ }^{*}$ Autor a quien debe ser dirigida la correspondencia.
}

Recibido Nov. 22, 2013; Aceptado Ene. 29, 2014; Versión final recibida Mar. 14, 2014

\begin{abstract}
Resumen
Este artículo propone un nuevo esquema de filtro híbrido adaptativo en cascada basado en los algoritmos de mínimos cuadrados promediados y recursivo de mínimos cuadrados para arreglos lineales uniformes de antenas para generar lóbulos de radiación. Los bloques adaptativos individuales usados para formar el nuevo filtro son interconectados a través del factor de arreglo lineal para simular un arreglo virtual de antenas y fijar la dirección deseada del lóbulo principal. La formulación completa del nuevo filtro se detalla y su capacidad de minimizar los efectos debidos a señales de interferencia y ruido blanco gaussiano aditivo es evaluada mediante simulación y comparada con otros filtros. Los resultados de la simulación mostraron que la rapidez de convergencia y las características de filtrado del nuevo modelo son mejores o similares a las de aquellos que lo constituyen. Se concluye que el nuevo esquema puede generar el lóbulo principal hacia la dirección de la señal deseada.
\end{abstract}

Palabras clave: arreglo lineal de antenas, filtros adaptativos en cascada, filtros híbridos, lóbulo de radiación, minimización de interferencias

\section{Adaptive Hybrid Filter in Cascade for Uniform Linear Antenna Arrays}

\begin{abstract}
This paper proposes a new adaptive hybrid filter scheme based on the least mean square and the recursive least square algorithms for uniform linear antenna arrays to generate radiation beam patterns. The individual adaptive blocks used to form the new filter are interconnected by the linear array factor to simulate a virtual antenna array and to set the desired direction of the main beam pattern. The completed formulation of the new filter is detailed and its capability to minimize interference signals and adaptive white gaussian noise is evaluated by simulation and compared with other filters. The simulation results showed that the convergence speed and the filtering characteristics of the new model are better or similar to those that make it. In conclusion, the new scheme can generate the main beam pattern to the desired signal direction.
\end{abstract}

Keywords: linear antenna arrays, cascade adaptive filters, hybrid filters, radiation beam pattern, interference minimization 


\section{INTRODUCCIÓN}

La recepción de señales basada en el uso de arreglo de sensores se considera una solución altamente beneficiosa en diversos problemas de detección y estimación de señales corruptas por ruido u otras señales de información no deseadas en un sistema de comunicaciones, esto debido a que un arreglo de elementos ofrece una mayor capacidad en la determinación de la directividad y supera las limitaciones relacionadas con el ancho de banda en la recepción o transmisión ocasionada por un solo elemento. Por esta razón, los arreglos de antenas adaptativos y el diseño de algoritmos de la misma naturaleza son actualmente objeto de una extensa investigación como un medio para reducir la vulnerabilidad de la recepción de las señales deseadas en presencia de ruido del ambiente o interferencias (señales no deseadas), a partir de otras fuentes en los sistemas de comunicaciones. En la actualidad debido a la alta capacidad de procesamiento de los sistemas computacionales es posible aprovechar por completo la teoría del procesamiento estadístico de señales, primordialmente su estimación y detección, además de los conceptos de la teoría de control para el desarrollo de sistemas que automáticamente responden al cambio de las señales en su entorno, conocidos como sistemas adaptativos o adaptables.

El filtrado de señales tanto en el dominio de espacio como en el de frecuencia en sistemas de comunicaciones se lo puede realizar por medio de estos sistemas adaptables, conformados básicamente por un arreglo de antenas y un procesador adaptativo, reduciendo así la sensibilidad del sistema a la recepción de ruido y/o señales no deseadas provenientes de fuentes de interferencia. El concepto de antena inteligente, en sí implica el hecho de que la calibración de los pesos puede hacerse adaptativa, por lo tanto, un conjunto de procesadores adaptativos filtran las señales procedentes de los sensores y generan un lóbulo de radiación principal a diferentes direcciones en las que se requiere una comunicación. Los pesos del sistema pueden ser ajustados automáticamente mediantes técnicas simples de adaptabilidad basadas en algoritmos de Mínimos Cuadrados Promediados (MCP) (Widrow y Stearns, 1985) o en el algoritmo Recursivo de Mínimos Cuadrados (RMC) (Sayed, 2003). Durante el proceso de adaptación una señal piloto se inyecta simulando una señal recibida desde una dirección deseada. Esto permite que el arreglo pueda ser entrenado de tal manera que su patrón de directividad genere un lóbulo principal en la dirección de observación previamente especificada. Al mismo tiempo, el sistema de procesamiento del arreglo puede rechazar cualquier ruido incidente o señales no requeridas, cuyas direcciones de propagación son diferentes a la de la dirección deseada, esto es posible debido a la formación de lóbulos nulos apropiados en el patrón de directividad de la antena. El arreglo se adapta por sí mismo para formar un lóbulo principal con su dirección y ancho de banda determinados por la señal piloto, y por consiguiente, así rechazar las señales o ruidos que se producen fuera del lóbulo principal en base al principio del Error Mínimo Cuadrático Promediado (EMCP).

Dentro de la literatura se han propuesto durante los últimos 15 años varios algoritmos y estructuras adaptativas enfocados al arreglo de antenas para mejorar su velocidad de convergencia, capacidad de estimación y minimización de ruido. En Liang y Chin (2000) se proponen dos algoritmos nuevos llamados algoritmo MCP Coherente (MCPC) y MCP Coherente-Normalizado (MCP-CN) en los cuales se restringe algunos de los componentes de la señal de salida del filtro para que permanezcan en fase con la señal de referencia en cada iteración, permitiendo así una mayor rapidez de convergencia utilizando el mismo tamaño de paso que su contraparte sencilla MCP. Otro tipo de sistemas que se estudia para los arreglos adaptativos es el de tipo híbrido, como por ejemplo el propuesto por Watanabe y Kohno (2000) cuyo esquema se basa en la combinación de un algoritmo MCP con la Transformada de Fourier Discreta (TFD). El objetivo es mejorar la actualización instantánea de los pesos del algoritmo MCP en cada iteración mediante una estimación inicial de sus valores en base a la TFD.

Debido a que los sistemas de comunicaciones y especialmente los inalámbricos deben ser capaces de servir a un gran número de suscriptores y por tanto suplir la demanda por una alta tasa de trasmisión de datos, algunas técnicas combinan la División Multiplexada Ortogonal de Frecuencia (DMOF) (Tuan et al., 2002) con el algoritmo RMC. Una ventaja de esta técnica es que la modulación y demodulación se lo hace en el dominio discreto usando la TFD, la cual se implementa fácilmente usando la Transformada Rápida de Fourier (TRF). Otros enfoques en el desarrollo de sistemas adaptativos a ser utilizados en arreglos de antenas permiten diseñar los algoritmos denominados robustos como el propuesto por Shubair (2005), donde el vector inicial de los pesos a ser utilizados en el algoritmo MCP es primero calculado por medio del algoritmo de Inversión de la Matriz de Muestras (IMM). Éste cálculo determina una estimación inicial óptima de los pesos, de tal manera que antes de empezar el proceso de adaptación del algoritmo MCP, éste ya produce un lóbulo de radiación apuntando aproximadamente hacia la dirección deseada. Algunos algoritmos nuevos como el de Mínimos Promediados de Norma Combinada (MPNC) (Shubair y Jimaa, 2007) se enfocan en minimizar funciones de costos formuladas en base al método de Alisado Exponencial Simple (AES), en el cual se involucra el error elevado a la segunda y cuarta potencia proveniente del algoritmo 
MCP como datos de entrada a la ecuación AES de tal manera que se llega a una convergencia más rápida que la del algoritmo MCP sencillo.

Recientemente, algunos algoritmos basados en técnicas numéricas e inteligencia artificial han sido diseñados. En Shi et al. (2012) se propone un nuevo diseño basado en algoritmos genéticos y funciones ortogonales para calcular la fase y amplitud de los elementos de excitación del arreglo lineal. En este concepto se reduce el número de variables involucradas en el algoritmo y se acelera su convergencia. En el nuevo método propuesto por Acevedo et al. (2012) se mejora la diversidad espacial al implementar y resolver un sistema de ecuaciones lineales que representan la magnitud de cada lóbulo lateral del patrón de radiación, la solución del sistema de ecuaciones mejora la intensidad tanto de los lóbulos secundarios como la del lóbulo principal en la dirección de la señal de información deseada. El esquema basado en redes neuronales y propuesto por Fernández et al. (2013) estima la dirección de arribo en arreglos lineales mediante el diseño de un sistema inteligente de dos etapas, una de detección y otra de estimación. Al dividir el proceso de aprendizaje de la red neuronal en dos bloques se reduce el tiempo de estimación total y se mejora la calidad de generación del lóbulo principal de radiación. Algunos arreglos lineales son utilizados como sistemas complementarios a sistemas de radares (Yang, 2013) y se diseñan en base al criterio del EMCP y la relación señal a ruido más interferencia (RSRI). El primer esquema calcula los pesos del sistema utilizando solamente el arreglo lineal, mientras que en el segundo se calculan incluyendo las antenas del radar. En esta nueva propuesta se mejora la ganancia del lóbulo principal y se minimiza el error en la ubicación de los nulos del patrón de radiación. El modelo de antena inteligente propuesto por Rahmani et al. (2014) considera un nuevo diseño de arreglos lineales basado en la eliminación de la señal piloto de entrenamiento del algoritmo adaptativo. Se considera la utilización de técnicas de sub-arreglos y se define una región de incertidumbre para estimar datos de entrada de aprendizaje al sistema, esto permite el cálculo del vector de pesos total del sistema y minimiza el error de precisión que podría ser ocasionado por el Vector del Arreglo Lineal (VAL) (Kraus y Marheka, 2002).

En este artículo se propone un esquema híbrido en cascada cuyo principal objetivo es generar lóbulos de radiación nulos en arreglos lineales de antenas, minimizando así los efectos de las señales de interferencia y la contaminación por Ruido Blanco Gaussiano Aditivo (RBGA) en los sistemas de comunicaciones. El esquema propuesto se basa en la utilización de un filtro adaptativos MCP como su etapa de pre-filtrado conectado en serie a un filtro adaptativo RMC a través de un VAL, permitiendo así un doble proceso de filtrado. Los resultados experimentales muestran la rapidez de convergencia y por tanto el número de iteraciones requerida frente a los sub-filtros MCP y RMC simples que constituyen el filtro híbrido. Se prueba su capacidad de filtrado para diferentes arreglos de antenas y su habilidad de generar el lóbulo de radiación principal hacia el Ángulo de Arribo Deseado (AAD). Finalmente, el análisis del costo computacional determina la cantidad de recursos utilizados en su proceso de cálculo frente a otros esquemas en cascada (Srar y Chung, 2009; Orozco et al, 2013) que utilizan filtros MCP y RMC. A continuación se detalla la organización del artículo. Primero, se describe la formulación completa del esquema propuesto y su convergencia a la solución de Wiener (Diniz, 2013) seguido del análisis matemático de su costo computacional. Segundo, se muestran los resultados obtenidos de la simulación por computadora, los valores numéricos del costo computacional y finalmente se concluye el artículo. En las conclusiones se establece que el nuevo esquema converge a una solución óptima igual o mejor que los filtros individuales que lo constituyen, minimiza los efectos de las señales de interferencia y del RGBA. Su costo computacional es inferior a otros esquemas de características similares y se observa que el filtro genera el lóbulo principal hacia la dirección de arribo de la señal deseada de información.

\section{ESQUEMA PROPUESTO}

En esta sección se proporciona una descripción detallada de la formulación del modelo híbrido propuesto, además de una estimación del costo computacional.

\section{Formulación del modelo}

La figura 1, muestra el diagrama de bloques del arreglo lineal híbrido adaptativo de $\mathrm{N}$-elementos isotrópicos propuesto. El bloque de entrada o pre-filtrado está conformado por un filtro transversal adaptativo con un algoritmo MCP, seguido por un segundo filtro en serie o cascada con un algoritmo RMC, siendo este el bloque principal que genera el lóbulo mayor en el patrón de radiación del arreglo lineal hacia el AAD. Ambos sub-filtros se interconectan por medio del VAL $(F)$ de la señal que se desea estimar. Este vector descompone la señal proveniente del primer bloque en diferentes componentes reales e imaginarios, simulando así un arreglo lineal de $\mathrm{M}$ elementos isotrópicos. Adicionalmente, el VAL permite fijar la dirección del lóbulo de radiación en la dirección deseada para recepción en el bloque RMC. 


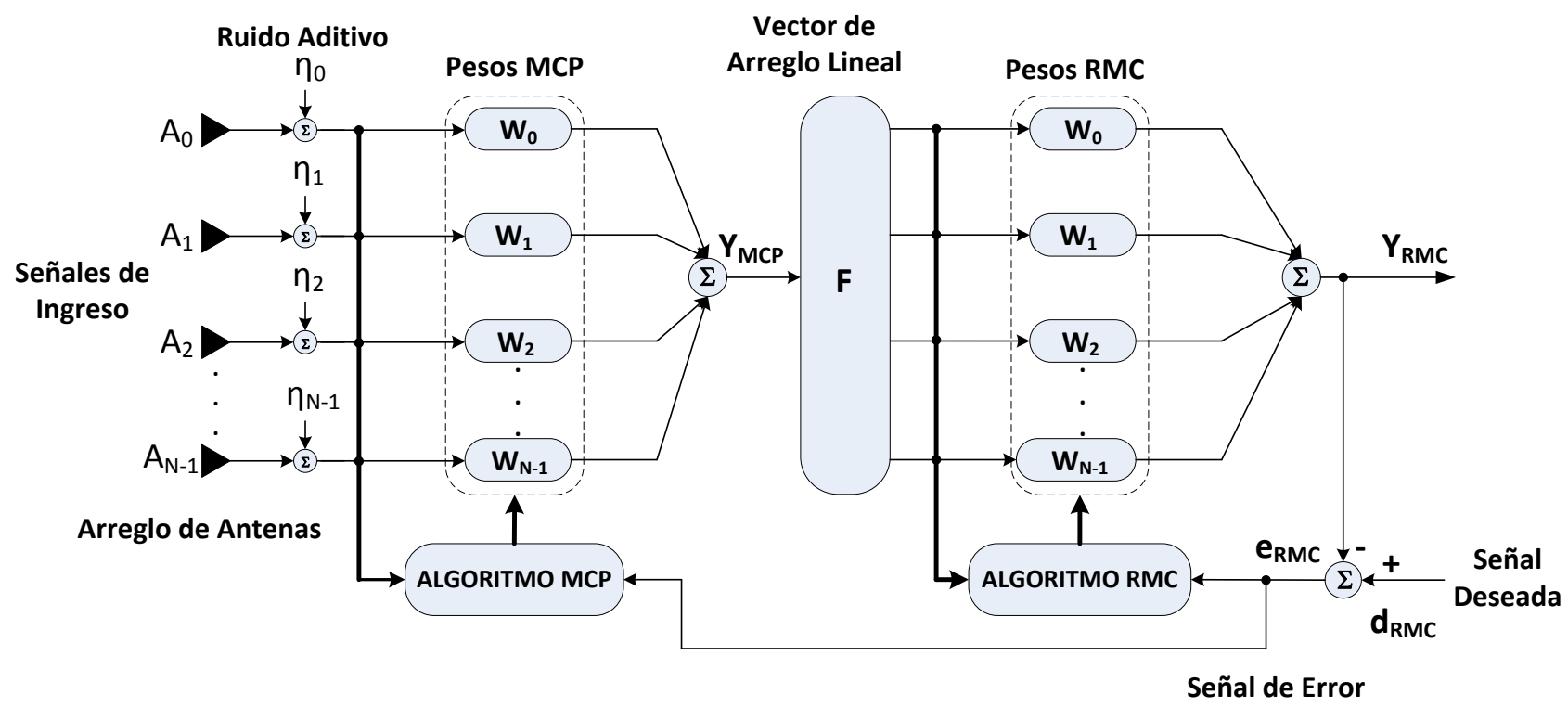

Fig. 1: Esquema híbrido adaptativo HCNEC

Debido a que ambos filtros ejecutan las iteraciones al mismo tiempo y dependen de la misma señal de error generada en el bloque RMC, el filtro ha sido denominado como filtro Híbrido en Cascada No independiente con Error Común (HCNEC). Las ecuaciones del modelo híbrido son desarrolladas en base al combinador lineal utilizado en la salida de cada etapa del sistema. Si se considera que todas las iteraciones han sido ejecutadas en el filtro de entrada por el algoritmo MCP, entonces su salida se determinada como

$\mathrm{y}_{\mathrm{MCP}}=\mathrm{W}_{\mathrm{MCP}}^{\mathrm{H}} \mathrm{X}_{\mathrm{MCP}}=\mathrm{X}_{\mathrm{MCP}}^{\mathrm{H}} \mathrm{W}_{\mathrm{MCP}}$

El operador \{\}$^{H}$ en la ecuación (1) representa la transpuesta Hermitiana, mientras que $\mathrm{W}_{\mathrm{MCP}}$ y $\mathrm{X}_{\mathrm{MCP}}$ representan el vector de pesos y entrada del bloque de pre-filtrado $\mathrm{MCP}$, respectivamente. Aunque el bloque MCP no genera su propia señal de error, no obstante los valores óptimos de sus pesos pueden obtenerse utilizando la señal de error proveniente del bloque RMC que es común para ambos sub-filtros. La estimación de los valores óptimos de los pesos que minimizan el error cuadrado promediado (ECP) del algoritmo MCP se basa en el principio de ortogonalidad, de tal manera que la respuesta al impulso del filtro sea optimizada. Si se considera que la señal de error de pos-filtrado $e_{\mathrm{RMC}}$ es no correlacionada con el vector de la señal de entrada al esquema de filtrado híbrido, entonces

$$
\begin{aligned}
& E\left\{e_{R M C} X_{M C P}\right\}=0 \\
& E\left\{\left(d_{R M C}-y_{R M C}\right) X_{M C P}\right\}=0 \\
& E\left\{d_{R M C} X_{M C P}\right\}-E\left\{X_{M C P} y_{R M C}\right\}=0
\end{aligned}
$$

El primer término del lado izquierdo de la ecuación (4) representa el vector columna de correlación cruzada $P_{M C P}$ entre la señal deseada dRMC y el vector de entrada al filtro MCP, mientras que la salida del bloque RMC se define como $\mathrm{y}_{\mathrm{RMC}}=\mathrm{W}_{\mathrm{RMC}}^{\mathrm{H}} \mathrm{X}_{\mathrm{RMC}}=\mathrm{X}_{\mathrm{RMC}}^{\mathrm{H}} \mathrm{W}_{\mathrm{RMC}}$, donde $\mathrm{W}_{\mathrm{RMC}}$ y $\mathrm{X}_{\mathrm{RMC}}$ son su vector de pesos y entrada, por lo tanto la ecuación (4) se expresa como

$P_{M C P}-E\left\{X_{M C P} X_{R M C}^{H} W_{R M C}^{*}\right\}=0$

El operador \{\}$^{*}$ en la ecuación (5) representa el valor óptimo del peso. Ahora, si se sustituye la ecuación (1) y siendo el vector VAL $(F)$ el factor que permite obtener la señal de entrada al bloque RMC al multiplicar y 
descomponer la señal de salida del filtro $\mathrm{MCP}$, es decir $\mathrm{X}_{\mathrm{RMC}}=\mathrm{Fy}_{\mathrm{MCP}}$, entonces la ecuación (5) puede ser escrita como

$$
\begin{aligned}
& P_{M C P}-E\left\{X_{M C P}\left(F y_{M C P}\right)^{H} W_{R M C}^{*}\right\}=0 \\
& P_{M C P}-E\left\{X_{M C P}\left(F\left(W_{M C P}^{H}\right)^{*} X_{M C P}\right)^{H} W_{R M C}^{*}\right\}=0 \\
& P_{M C P}-E\left\{X_{M C P} X_{M C P}^{H} W_{M C P}^{*} F^{H} W_{R M C}^{*}\right\}=0 \\
& P_{M C P}-E\left\{X_{M C P} X_{M C P}^{H} W_{M C P}^{*} F^{H} W_{R M C}^{*}=0\right.
\end{aligned}
$$

Si se observa con detalle la ecuación (9), el factor $E\left\{X_{M C P} X_{M C P}^{H}\right\}$ es la matriz de auto correlación correspondiente al vector de entrada al filtro MCP, denotado por $\mathrm{R}$, por consiguiente los pesos óptimos de la etapa de pre-filtrado correspondiente a este algoritmo se calculan como

$$
\begin{aligned}
& \mathrm{P}_{\mathrm{MCP}}-\mathrm{R}_{\mathrm{MCP}} \mathrm{W}_{\mathrm{MCP}}^{*} \mathrm{~F}^{\mathrm{H}} \mathrm{W}_{\mathrm{RMC}}^{*}=0 \\
& \mathrm{~W}_{\mathrm{MCP}}^{*}=\left(\mathrm{R}_{\mathrm{MCP}}\right)^{-1} \mathrm{P}_{\mathrm{MCP}}\left(\mathrm{F}^{\mathrm{H}} \mathrm{W}_{\mathrm{RMC}}^{*}\right)^{-1}
\end{aligned}
$$

Con la intención de simplificar aún más la expresión obtenida en la ecuación (11), se define un nuevo vector de correlación cruzada $\mathrm{P}_{\mathrm{MCP}}^{\prime}=\mathrm{P}_{\mathrm{MCP}}\left(\mathrm{F}^{\mathrm{H}} \mathrm{W}_{\mathrm{RMC}}^{*}\right)$, por lo tanto la expresión final del vector correspondiente a los pesos óptimos del sub-filtro MCP, puede ser escrita como

$$
\mathrm{W}_{\mathrm{MCP}}^{*}=\left(\mathrm{R}_{\mathrm{MCP}}\right)^{-1} \mathrm{P}_{\mathrm{MCP}}^{\prime}
$$

La ecuación (12) converge a la solución de Wiener (Diniz, 2013), y depende del valor óptimo de los pesos del bloque RMC, así como del VAL. Este hecho se debe a que durante la ejecución simultánea de ambos algoritmos, el vector de pesos RMC converge más rápido a una solución óptima que los correspondientes al vector de pesos del algoritmo MCP. Adicionalmente a esta característica propia del algoritmo, durante el proceso inicial de ejecución, los pesos del vector $W_{M C P}$ no se calculan sino después de que se ha realizado la primera iteración del algoritmo RMC para determinar los pesos correspondientes a su vector $\mathrm{W}_{\mathrm{RMC}}$, ya que los primeros dependen del error que éste sub-filtro genere en la primera iteración y en las posteriores. Ahora, el vector de pesos óptimos correspondiente al bloque RMC se determina siguiendo un procedimiento similar al del esquema de pre-filtrado MCP, por consiguiente

$$
\begin{aligned}
& E\left\{e_{\mathrm{RMC}} \mathrm{X}_{\mathrm{RMC}}\right\}=0 \\
& \mathrm{E}\left\{\left(\mathrm{d}_{\mathrm{RMC}}-\mathrm{y}_{\mathrm{RMC}}\right) \mathrm{X}_{\mathrm{RMC}}\right\}=0 \\
& \mathrm{E}\left\{\mathrm{d}_{\mathrm{RMC}} \mathrm{X}_{\mathrm{RMC}}\right\}-\mathrm{E}\left\{\mathrm{X}_{\mathrm{RMC}} \mathrm{y}_{\mathrm{RMC}}\right\}=0
\end{aligned}
$$

Considerando el hecho de que la entrada al bloque RMC se determinada por $\mathrm{X}_{\mathrm{RMC}}=\mathrm{Fy}_{\mathrm{MCP}}$, entonces el primer término del lado izquierdo de la ecuación (15) puede ser expresado en términos del vector columna de correlación cruzada $\mathrm{P}$ y de los pesos óptimos del bloque MCP, de tal manera que

$$
E\left\{d_{R M C} X_{R M C}\right\}=E\left\{d_{R M C} y_{M C P} F\right\}
$$


$E\left\{d_{\text {RMC }} X_{\text {RMC }}\right\}=E\left\{d_{\text {RMC }} X_{M C P}^{H}\right\} W_{M C P}^{*} F$

$E\left\{d_{\mathrm{RMC}} \mathrm{X}_{\mathrm{RMC}}\right\}=\mathrm{P}_{\mathrm{MCP}}^{\mathrm{H}} \mathrm{W}_{\mathrm{MCP}}^{*} \mathrm{~F}$

Siguiendo un procedimiento similar al utilizado en el primer término del lado izquierdo de la ecuación (15), el segundo término puede ser expresado en términos del vector de pesos del sub-filtro MCP, el vector VAL y la matriz de auto-correlación de la señal de ingreso, por consiguiente

$$
\begin{aligned}
& \mathrm{E}\left\{\mathrm{X}_{\mathrm{RMC}} \mathrm{Y}_{\mathrm{RMC}}\right\}=\mathrm{E}\left\{\mathrm{X}_{\mathrm{RMC}} \mathrm{X}_{\mathrm{RMC}}^{\mathrm{H}} \mathrm{W}_{\mathrm{RMC}}^{*}\right\} \\
& E\left\{X_{R_{M C C}} y_{\text {RMC }}\right\}=E\left\{F\left(W_{M C P}^{H}\right)^{*} X_{M C P}\left[F\left(W_{M C P}^{H}\right)^{*} X_{M C P}\right]^{H} W_{R M C}^{*}\right\} \\
& E\left\{X_{R M C} y_{R M C}\right\}=E\left\{F\left(W_{M C P}^{H}\right)^{*} X_{M C P} X_{M C P}^{H} W_{M C P}^{*} F^{H} W_{R M C}^{*}\right\} \\
& E\left\{X_{R M C} Y_{R M C}\right\}=F\left(W_{M P C}^{H}\right)^{*} E\left\{X_{M C P} X_{M C P}^{H} W_{M C P}^{*} F^{H} W_{R M C}^{*}\right. \\
& \mathrm{E}\left\{\mathrm{X}_{\mathrm{RMC}} \mathrm{Y}_{\mathrm{RMC}}\right\}=\mathrm{F}\left(\mathrm{W}_{\mathrm{MCP}}^{\mathrm{H}}\right)^{*} \mathrm{R}_{\mathrm{PMC}} \mathrm{W}_{\mathrm{PMC}}^{*} \mathrm{~F}^{\mathrm{H}} \mathrm{W}_{\mathrm{RMC}}^{*}
\end{aligned}
$$

Sustituyendo las ecuaciones (18) y (23) en la ecuación (15), finalmente se obtiene la expresión de los pesos correspondientes al sub-filtro RMC, por tanto

$$
\begin{aligned}
& P_{M C P}^{H} W_{M C P}^{*} F-F\left(W_{M C P}^{H}\right)^{*} R_{M C P} W_{M C P}^{*} F^{H} W_{R M C}^{*}=0 \\
& F\left(W_{M C P}^{H}\right)^{*} R_{M C P} W_{M C P}^{*} F^{H} W_{M C P}^{*}=P_{M C P}^{H} W_{M C P}^{*} F \\
& W_{R M C}^{*}=\left(F\left(W_{M C P}^{H}\right)^{*} R_{M C P} W_{M C P}^{*} F^{H}\right)^{-1} P_{M C P}^{H} W_{M C P}^{*} F
\end{aligned}
$$

Una vez más, si se definen dos nuevos vectores de correlación cruzada $P_{\mathrm{RMC}}^{\prime}$ y auto correlación $\mathrm{R}_{\mathrm{RMC}}^{\prime}$ para la ecuación (26), se llega a la solución óptima de Wiener, por $\mathrm{W}_{\mathrm{RMC}}^{*}$ consiguiente

$$
\begin{aligned}
& \mathrm{R}_{\mathrm{RMC}}^{\prime}=\mathrm{F}\left(\mathrm{W}_{\mathrm{MCP}}^{\mathrm{H}}\right)^{*} \mathrm{R}_{\mathrm{MCP}} \mathrm{W}_{\mathrm{MCP}}^{*} \mathrm{~F}^{\mathrm{H}} \\
& \mathrm{P}_{\mathrm{RMC}}^{\prime}=\mathrm{P}_{\mathrm{MCP}}^{\mathrm{H}} \mathrm{W}_{\mathrm{MCP}}^{*} \mathrm{~F} \\
& \mathrm{~W}_{\mathrm{RMC}}^{*}=\left(\mathrm{R}_{\mathrm{RMC}}^{\prime}\right)^{-1} \mathrm{P}_{\mathrm{RMC}}^{\prime}
\end{aligned}
$$

En la figura 1 la señal de error $e_{\mathrm{RMC}}=\varepsilon$ se calcula como

$$
\varepsilon=\mathrm{d}_{\mathrm{RMC}}-\mathrm{y}_{\mathrm{RMC}}
$$


Si se sustituyen $\mathrm{y}_{\mathrm{RMC}}=\mathrm{X}_{\mathrm{RMC}}^{\mathrm{H}} \mathrm{W}_{\mathrm{RMC}}, \mathrm{X}_{\mathrm{RMC}}=\mathrm{Fy} \mathrm{y}_{\mathrm{MCP}}$ y la ecuación (1) en la ecuación (31), entonces es posible expresar la ecuación de error en términos del vector de entrada al filtro híbrido y de los pesos correspondientes a cada sub-filtro, por tanto

$$
\begin{aligned}
& \varepsilon=\mathrm{d}_{\mathrm{RMC}}-\mathrm{X}_{\mathrm{RMC}}^{\mathrm{H}} \mathrm{W}_{\mathrm{RMC}} \\
& \varepsilon=\mathrm{d}_{\mathrm{RMC}}-\mathrm{y}_{\mathrm{MCP}} \mathrm{F}^{\mathrm{H}} \mathrm{W}_{\mathrm{RMC}} \\
& \varepsilon=\mathrm{d}_{\mathrm{RMC}}-\mathrm{X}_{\mathrm{MCP}}^{\mathrm{H}} \mathrm{W}_{\mathrm{MCP}} \mathrm{F}^{\mathrm{H}} \mathrm{W}_{\mathrm{RMC}}
\end{aligned}
$$

Ahora, al elevar al cuadrado la ecuación (33) y definir un vector de pesos común dado por $\mathrm{W}_{\mathrm{CH}}=\mathrm{W}_{\mathrm{MCP}} \mathrm{F}^{\mathrm{H}} \mathrm{W}_{\mathrm{RMC}}$, se obtiene la ecuación de error cuadrado promediado (ECP), $\varepsilon^{2}$ del esquema híbrido

$$
\begin{aligned}
& \varepsilon=d_{R M C}-X_{M C P}^{H} W_{C H} \\
& \varepsilon^{2}=d_{R M C}^{2}-2 d_{R M C} X_{M C P}^{H} W_{C H}+W_{C H}^{H} X_{M C P} X_{M C P}^{H} W_{C H}
\end{aligned}
$$

El subíndice $\mathrm{CH}$ en el vector de pesos $\mathrm{W}$ se refiere al modelo en cascada híbrido completo. Si se asume que $\varepsilon, d_{R M C}$ y $X_{M C P}$ son estadísticamente estacionarios y se toma el valor esperado de la ecuación (35), se obtiene finalmente la expresión final del ECP

$$
\begin{aligned}
& E\left\{\varepsilon^{2}\right\}=E\left\{d_{R M C}^{2}\right\}-2 E\left\{d_{R M C} X_{M C P}^{H}\right\} W_{C H}+W_{C H}^{H} E\left\{X_{M C P} X_{M C P}^{H} W_{C H}\right. \\
& \psi=E\left\{d_{R M C}^{2}\right\}-2 P_{R M C}^{H} W_{C H}+W_{C H}^{H} R_{M C P} W_{C H}
\end{aligned}
$$

En los procesos adaptativos, es indispensable determinar el valor de los pesos óptimos del filtro que calculan el error mínimo cuadrado promediado (EMCP). Uno de los procedimientos matemáticos que permite obtenerlo es el método del gradiente, designado por $\nabla$, que se calcula al derivar la ecuación (37) con respecto a cada uno de los pesos $\mathrm{W}_{\mathrm{CH}}$ que conforman el filtro, por tanto

$$
\nabla=2 \mathrm{R}_{\mathrm{MCP}} \mathrm{W}_{\mathrm{CH}}-2 \mathrm{P}_{\mathrm{RMC}}
$$

Aunque la ecuación (38) expresa un concepto sencillo; sin embargo, es importante recordar que el vector de pesos global del sistema híbrido está conformado por los pesos de los sub-filtros MCP y RMC que constituyen todo el esquema. Para obtener el EMCP, el vector de pesos $\mathrm{W}_{\mathrm{CH}}$ debe converger a su valor óptimo $\mathrm{W}_{\mathrm{CH}}^{*}$, en donde el gradiente es cero,

$0=2 \mathrm{R}_{\mathrm{MCP}} \mathrm{W}_{\mathrm{CH}}^{*}-2 \mathrm{P}_{\mathrm{RMC}}$

Asumiendo que la matriz de auto-correlación es una matriz no singular, el valor óptimo de los pesos se determina finalmente por

$$
\begin{aligned}
& \mathrm{R}_{\mathrm{MCP}} \mathrm{W}_{\mathrm{CH}}^{*}=\mathrm{P}_{\mathrm{RMC}} \\
& \mathrm{W}_{\mathrm{CH}}^{*}=\left(\mathrm{R}_{\mathrm{MCP}}\right)^{-1} \mathrm{P}_{\mathrm{RMC}}
\end{aligned}
$$


Esta última expresión es una ecuación de Wiener en su forma matricial. Es importante notar que el vector de pesos óptimos depende de la matriz de auto-correlación del vector de entrada al filtro MCP y del vector de correlación cruzada del segundo filtro RMC. EI EMCP es finalmente obtenido sustituyendo la ecuación (41) en (37),

$$
\begin{aligned}
& \psi_{\text {min }}=E\left\{d_{\mathrm{RMC}}^{2}\right\}-2 \mathrm{P}_{\mathrm{RMC}}^{\mathrm{H}} \mathrm{W}_{\mathrm{CH}}^{*}+\left(\mathrm{W}_{\mathrm{CH}}^{\mathrm{H}}\right)^{*} \mathrm{R}_{\mathrm{MCP}} \mathrm{W}_{\mathrm{CH}}^{*} \\
& \psi_{\text {min }}=\mathrm{E}\left\{\mathrm{d}_{\mathrm{RMC}}^{2}\right\}-2 \mathrm{P}_{\mathrm{RMC}}^{\mathrm{H}}\left(\mathrm{R}_{\mathrm{MCP}}\right)^{-1} \mathrm{P}_{\mathrm{RMC}}+\left(\left(\mathrm{R}_{\mathrm{MCP}}\right)^{-1} \mathrm{P}_{\mathrm{RMC}}\right)^{-H} \mathrm{R}_{\mathrm{MCP}}\left(\mathrm{R}_{\mathrm{MCP}}\right)^{-1} \mathrm{P}_{\mathrm{RMC}} \\
& \psi_{\text {min }}=\mathrm{E}\left\{\mathrm{d}_{\mathrm{RMC}}^{2}\right\}-2 \mathrm{P}_{\mathrm{RMC}}^{\mathrm{H}}\left(\mathrm{R}_{\mathrm{MCP}}\right)^{-1} \mathrm{P}_{\mathrm{RMC}}+\mathrm{P}_{\mathrm{RMC}}^{\mathrm{H}}\left(\mathrm{R}_{\mathrm{MCP}}\right)^{-1} \mathrm{P}_{\mathrm{RMC}} \\
& \psi_{\min }=\mathrm{E}\left\{\mathrm{d}_{\mathrm{RMC}}^{2}\right\}-\mathrm{P}_{\mathrm{RMC}}^{\mathrm{H}}\left(\mathrm{R}_{\mathrm{MCP}}\right)^{-1} \mathrm{P}_{\mathrm{RMC}} \\
& \psi_{\min }=\mathrm{E}\left\{\mathrm{d}_{\mathrm{RMC}}^{2}\right\}-\mathrm{P}_{\mathrm{RMC}}^{\mathrm{H}} \mathrm{W}_{\mathrm{CH}}^{*}
\end{aligned}
$$

\section{Costo Computacional}

La actualización de los pesos de los bloques MCP y RMC se obtienen a través de los algoritmos que dan sus nombres, respectivamente. La convergencia hacia el valor óptimo se basa en minimizar el error cuadrado promediado (ECP), dado por

$$
\min _{w} E|d-x w|^{2}
$$

En donde $d$ es la señal piloto o deseada, $x$ es el vector correspondiente a la señal de ingreso y w el vector de pesos óptimo, el cual se calcula iterativamente por su algoritmo de gradiente MCP (Sayed, 2003) dado por

$$
\mathrm{w}_{\mathrm{i}}=\mathrm{w}_{\mathrm{i}-1}+\mu \mathrm{x}_{\mathrm{i}}^{*}\left[\mathrm{~d}(\mathrm{i})-\mathrm{x}_{\mathrm{i}} \mathrm{w}_{\mathrm{i}-1}\right] \text { Parai } \geq 0, \mathrm{w}_{-1}=\text { Valorinicial }
$$

mientras que, el segundo bloque calcula su vector óptimo por medio de su algoritmo recursivo RMC (Sayed, 2003) definido por

$$
\begin{aligned}
& P_{i}=\lambda^{-1}\left[P_{i-1} \frac{\lambda^{-1} P_{i-1} x_{i}^{*} x_{i} P_{i-1}}{1+\lambda^{-1} x_{i} P_{i-1} x_{i}^{*}}\right] \\
& w_{i}=w_{i-1}+P_{i} x_{i}^{*}\left[d(i)-x_{i} w_{i-1}\right]
\end{aligned}
$$

Con condición inicial $P_{-1}=\epsilon^{-1}$ I para un escalar positivo-pequeño $\in$ y un factor de olvido $0 \ll \lambda \leq 1$. La figura 2 , muestra el diagrama de flujos de la programación del algoritmo. En la ecuación (48) cada iteración requiere la evaluación del producto escalar $\mathrm{X}_{\mathrm{i}} \mathrm{W}_{\mathrm{i}-1}$, entre dos vectores de dimensión [1 $\mathrm{xM}$ y $[\mathrm{Mx} 1]$ para $\mathrm{M}$ elementos del arreglo. Este cálculo requiere $\mathrm{M}$ multiplicaciones complejas y $\mathrm{M}-1$ sumas complejas. Usando el hecho de que una multiplicación compleja requiere cuatro multiplicaciones reales y dos sumas reales, mientras que una suma compleja requiere dos sumas reales, entonces se requiere $4 \mathrm{M}$ multiplicaciones reales y $4 \mathrm{M}-2$ sumas reales. Realizando un análisis similar para cada cálculo de las ecuaciones (48), (49) y (50), es posible estimar el costo requerido del algoritmo híbrido. La Tabla 1 (Sayed, 2003) muestra un resumen del costo computacional estimado de los algoritmos MCP y RMC por iteración para datos complejos en términos del número de multiplicaciones, sumas y divisiones reales empleados en el cálculo. 


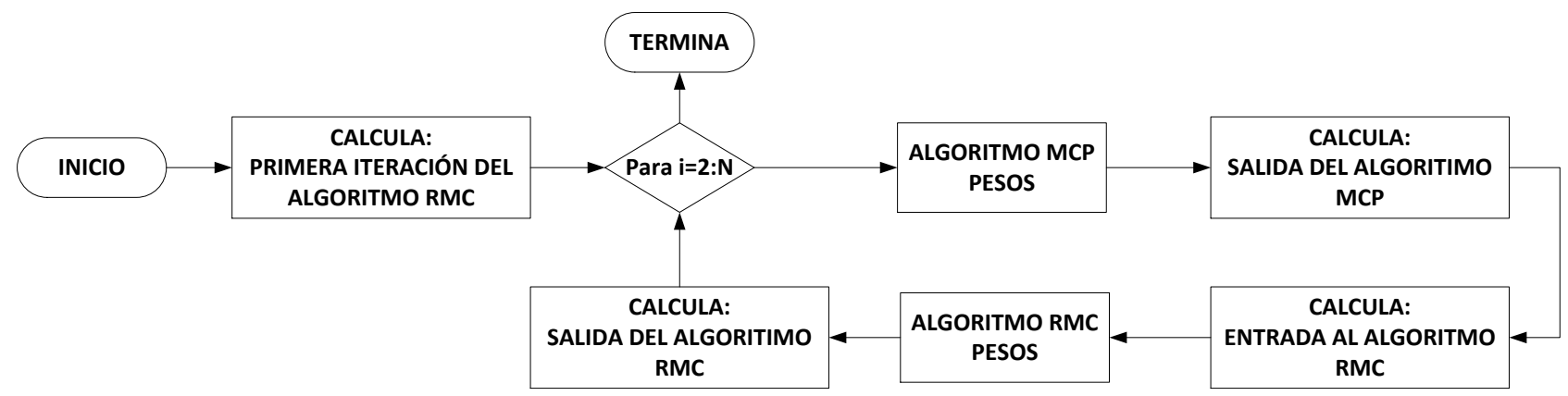

Fig. 2: Diagrama de flujo de la programación del esquema híbrido HCNEC

Tabla 1: Costo computacional estimado de los algoritmos MCP y RMC por iteración

\begin{tabular}{|c|c|c|c|c|c|c|c|}
\hline \multicolumn{4}{|c|}{ MCP } & \multicolumn{4}{|c|}{ RMC } \\
\hline Término & $x$ & + & 1 & Término & $x$ & + & 1 \\
\hline $\mathrm{X}_{\mathrm{i}} \mathrm{W}_{\mathrm{i}-1}$ & $4 \mathrm{M}$ & $4 M-2$ & & $\mathrm{X}_{\mathrm{i}} \mathrm{W}_{\mathrm{i}-1}$ & $4 \mathrm{M}$ & $4 \mathrm{M}-2$ & \\
\hline$d(i)-X_{i} W_{i-1}$ & & 2 & & $d(i)-X_{i} W_{i-1}$ & & 2 & \\
\hline$\mu\left[\mathrm{d}(\mathrm{i})-\mathrm{X}_{\mathrm{i}} \mathrm{W}_{\mathrm{i}-1}\right]$ & 2 & & & $\lambda^{-1} x_{i}^{*}$ & $2 \mathrm{M}$ & & \\
\hline$\mu X_{i}^{*}\left[d(i)-X_{i} W_{i-1}\right]$ & $4 \mathrm{M}$ & $2 \mathrm{M}$ & & $P_{i-1}\left(\lambda^{-1} x_{i}^{*}\right)$ & $4 M^{2}$ & $M(4 M-2)$ & \\
\hline $\mathrm{W}_{\mathrm{i}}$ & & $2 \mathrm{M}$ & & $x_{i} P_{i-1}\left(\lambda^{-1} x_{i}^{*}\right)$ & $4 M$ & $4 M-2$ & \\
\hline & & & & $1+x_{i} P_{i-1}\left(\lambda^{-1} x_{i}^{*}\right)$ & & 1 & \\
\hline & & & & $1 /\left[1+x_{i} P_{i-1}\left(\lambda^{-1} x_{i}^{*}\right)\right]$ & & & 1 \\
\hline & & & & $\left(\lambda^{-1} x_{i} P_{i-1} x_{i}^{*}\right) \cdot \frac{1}{1+x_{i} P_{i-1}\left(\lambda^{-1} x_{i}^{*}\right)}$ & 1 & & \\
\hline & & & & $\left(\lambda^{-1} \mathrm{P}_{\mathrm{i}-1} \mathrm{x}_{\mathrm{i}}^{*}\right) \times \frac{\lambda^{-1} \mathrm{x}_{\mathrm{i}} \mathrm{P}_{\mathrm{i}-1} \mathrm{x}_{\mathrm{i}}^{*}}{1+\lambda^{-1} \mathrm{x}_{\mathrm{i}} \mathrm{P}_{\mathrm{i}-1} \mathrm{x}_{\mathrm{i}}^{*}}$ & $2 \mathrm{M}$ & & \\
\hline & & & & $P_{i} x_{i}^{*}$ & & $2 \mathrm{M}$ & \\
\hline & & & & $P_{i} x_{i}^{*}\left[d(i)-X_{i} W_{i-1}\right]$ & $4 \mathrm{M}$ & $2 \mathrm{M}$ & \\
\hline & & & & $\mathrm{W}_{\mathrm{i}}$ & & $2 \mathrm{M}$ & \\
\hline Total & $8 M+2$ & $8 \mathrm{M}$ & & Total & $4 M^{2}+16 M+1$ & $4 M^{2}+12 M-1$ & 1 \\
\hline
\end{tabular}

Debido a que el error entre la señal de salida del filtro y la señal deseada se calcula exclusivamente en el algoritmo RMC del filtro HCNEC, entonces no se requieren las $4 \mathrm{M}$ primeras operaciones concernientes a la multiplicación y las dos primeras operaciones de las sumas 4M-2 y 2 del algoritmo MCP. La Tabla 2 muestra el costo computacional por iteración para el esquema HCNEC.

Tabla 2: Costo computacional estimado del esquema híbrido

\begin{tabular}{|c|c|c|c|}
\hline Esquema & \multicolumn{3}{|c|}{ Esquema HCNEC } \\
\hline Algoritmo & $\times$ & + & $/$ \\
\hline $\mathrm{MCP}$ & $4 \mathrm{M}+2$ & $4 \mathrm{M}$ & 1 \\
\hline $\mathrm{RMC}$ & $4 \mathrm{M}^{2}+16 \mathrm{M}+1$ & $4 \mathrm{M}^{2}+12 \mathrm{M}-1$ & 1 \\
\hline
\end{tabular}




\section{RESULTADOS}

Para evaluar el esquema híbrido propuesto, se realizaron simulaciones utilizando Matlab® y los siguientes parámetros:

i) Arreglos lineales de 4, 8, 12, 16 y 20 elementos isotrópicos.

ii) Distancia entre antenas $d=\square / 4^{*}$. (*Aquí $\square$ presenta la longitud de onda).

iii) Una señal de información banda base de $1 \mathrm{~Hz}$ y amplitud unitaria con ángulo de incidencia de 60 grados

iv) Una señal de interferencia de $2 \mathrm{~Hz}$ y amplitud 0.2 con un ángulo de incidencia de 45 grados.

v) Ruido Blanco Gaussiano Aditivo (RBGA) con varianza de 0.1 .

vi) Tamaño de paso del algoritmo MCP: $0.01 \leq \mu \leq 0.25$.

vii) Factor de olvido del algoritmo RMC: $\square \approx 1$.

viii) Cada simulación del algoritmo involucra 500 muestras.

La figura 3 muestra la señal de información banda base, la señal de interferencia y el RBGA utilizado para los arreglos lineales. En la figura 4 se muestra las dos primeras componentes real e imaginaria de la señal de información distorsionada por la señal de interferencia y contaminada por el RBGA para los arreglos de 4 hasta 20 elementos utilizados en la simulación. La figura 5 muestra las curvas de aprendizaje para diferentes modelos del filtro HCNEC para los esquemas con igual número de antenas reales y virtuales, es decir 4-4, 12-12 y 20-20. Para estos filtros, se observa que los valores de convergencia están alrededor de $-15 \mathrm{~dB},-20 \mathrm{~dB}$ y $-23 \mathrm{~dB}$, respectivamente; los cuales se encuentran por debajo de la relación señal a ruido (RSR) de 7dB. La RSR se define como la razón entre la potencia promedio de la señal de información y la potencia promedio del ruido en la entrada del arreglo y expresada en decibeles (dB), es decir $10 \log _{10}(\mathrm{RSR})$ (Haykin, 2001).

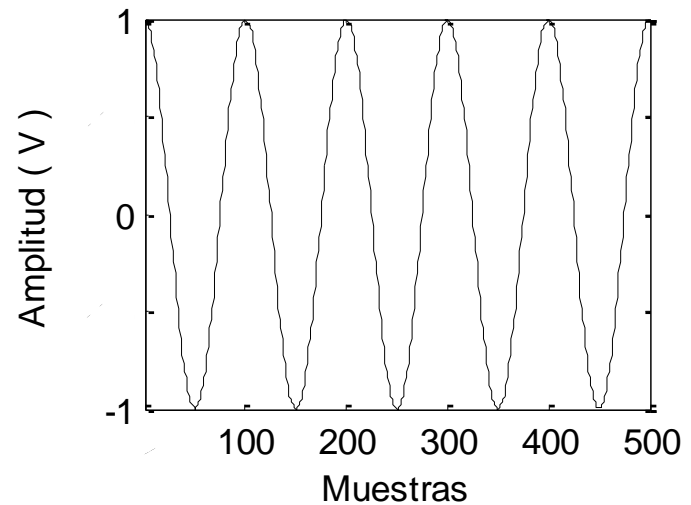

(a)

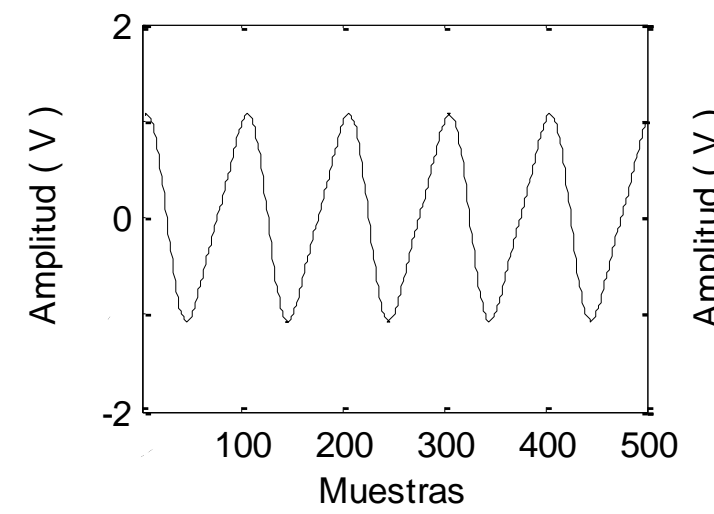

(c)

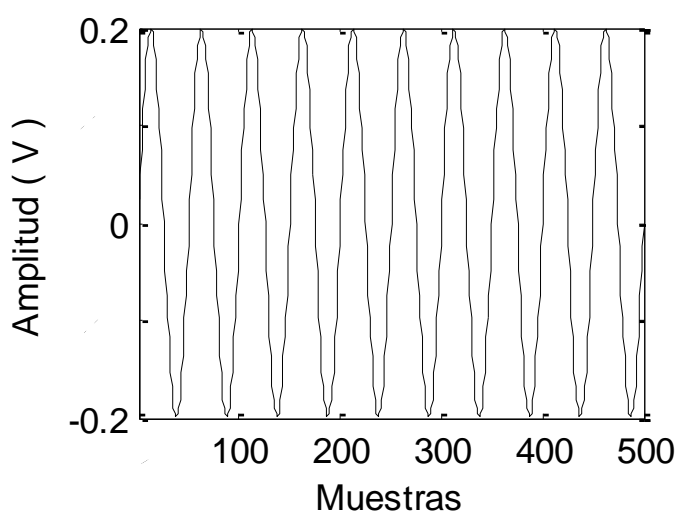

(b)

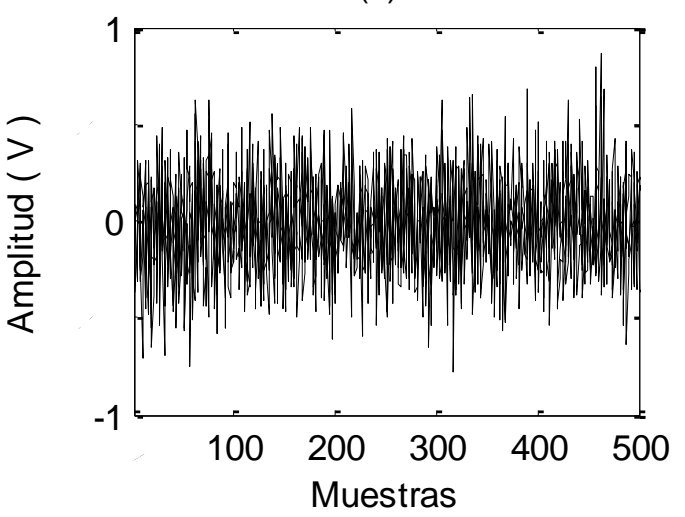

(d)

Fig. 3: (a) Señal de información, (b) Señal de interferencia, (c) Señal de información distorsionada por la señal de interferencia y (d) RGBA de varianza 0.1. 


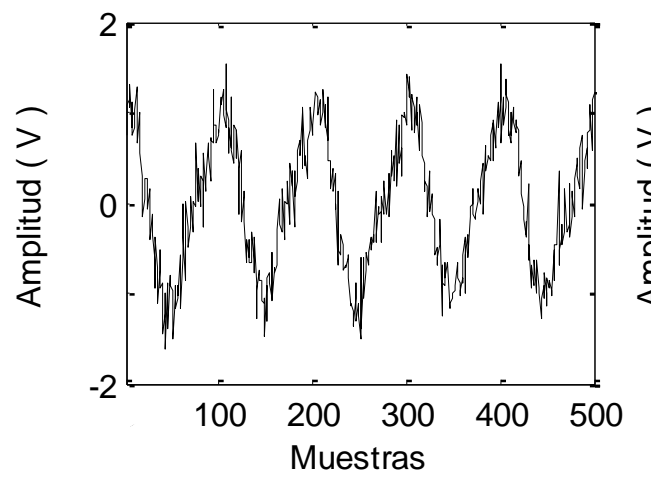

(a)

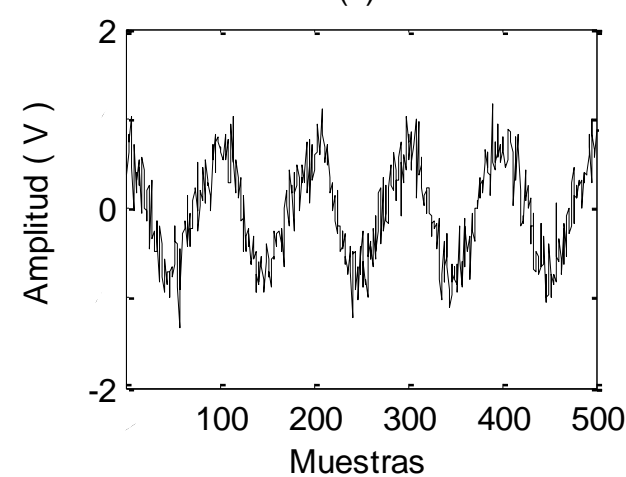

(c)

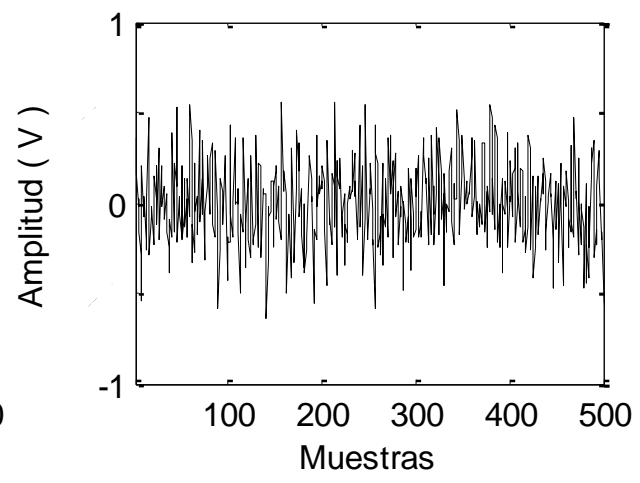

(b)

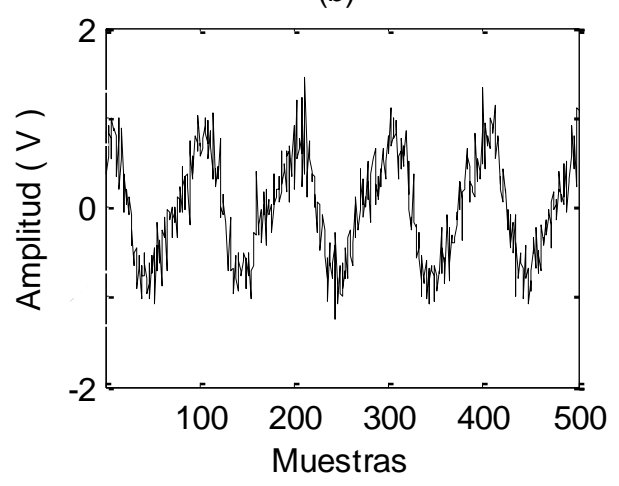

(d)

Fig. 4: (a) y (b) Componente real e imaginaria recibida por la primera antena, respectivamente; (c) y (d) Componente real e imaginaria recibida por la segunda antena, respectivamente.

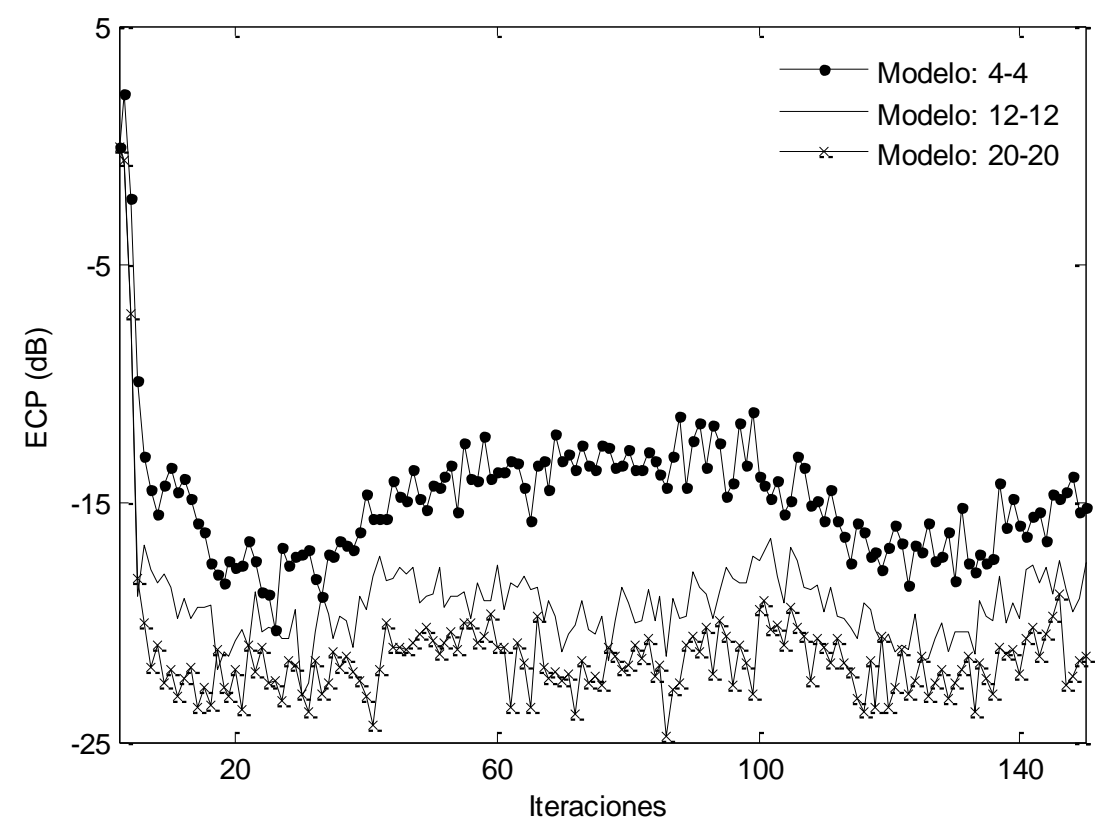

Fig. 5: Curva de ECP del algoritmo híbrido con igual número de antenas reales y virtuales.

De igual manera, la figura 6 muestra las curvas para los modelos con antenas reales y virtuales diferentes como son los esquemas: $4-20,8-20$ y $12-20$, tienen sus valores de convergencia muy cercanos a $-15 \mathrm{~dB}$, $18 \mathrm{~dB}$ y $-20 \mathrm{~dB}$, respectivamente. Las curvas de aprendizaje mostradas son curvas promedio correspondientes a 25 simulaciones para cada una, adicionalmente se muestran las primeras 150 iteraciones para efecto de una mejor visualización de los resultados. Ambas curvas fueron simuladas con un MCP- $\mu$ de 0.09 . 


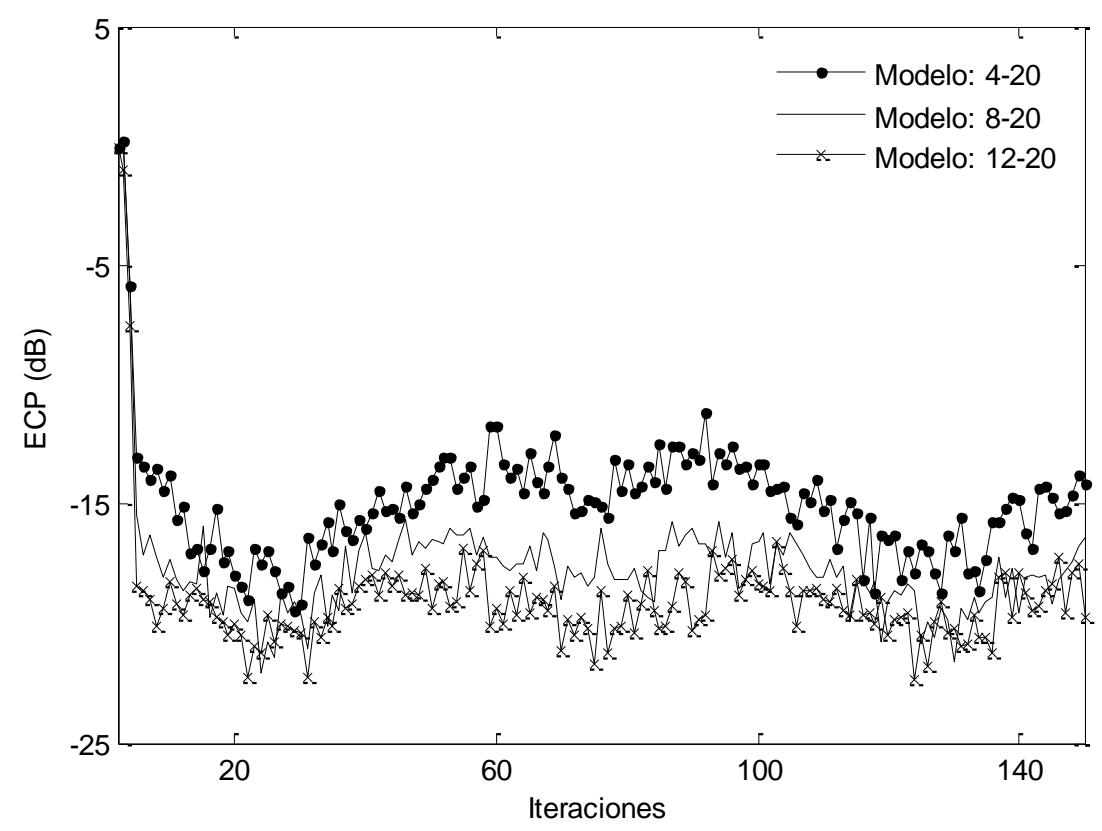

Fig. 6: Curva de ECP del algoritmo híbrido con diferente número de antenas reales y virtuales.

La figura 7 muestra una comparación entre las curvas de aprendizaje del esquema HCNEC 12-12 y los algoritmos MCP y RMC de 12 antenas y/o pesos utilizados en el diseño del modelo híbrido. Se puede apreciar que aunque el algoritmo híbrido utiliza un doble proceso de filtrado, esto no implica necesariamente un incremento en el número de iteraciones; incluso se converge a la solución óptima del problema de manera semejante al algoritmo MCP y mucho más rápido que el algoritmo RMC.

La figura 8 muestra el valor de aprendizaje estimado de diferentes esquemas HCNEC. Por conveniencia su valor ha sido expresado en unidades y no en decibeles.

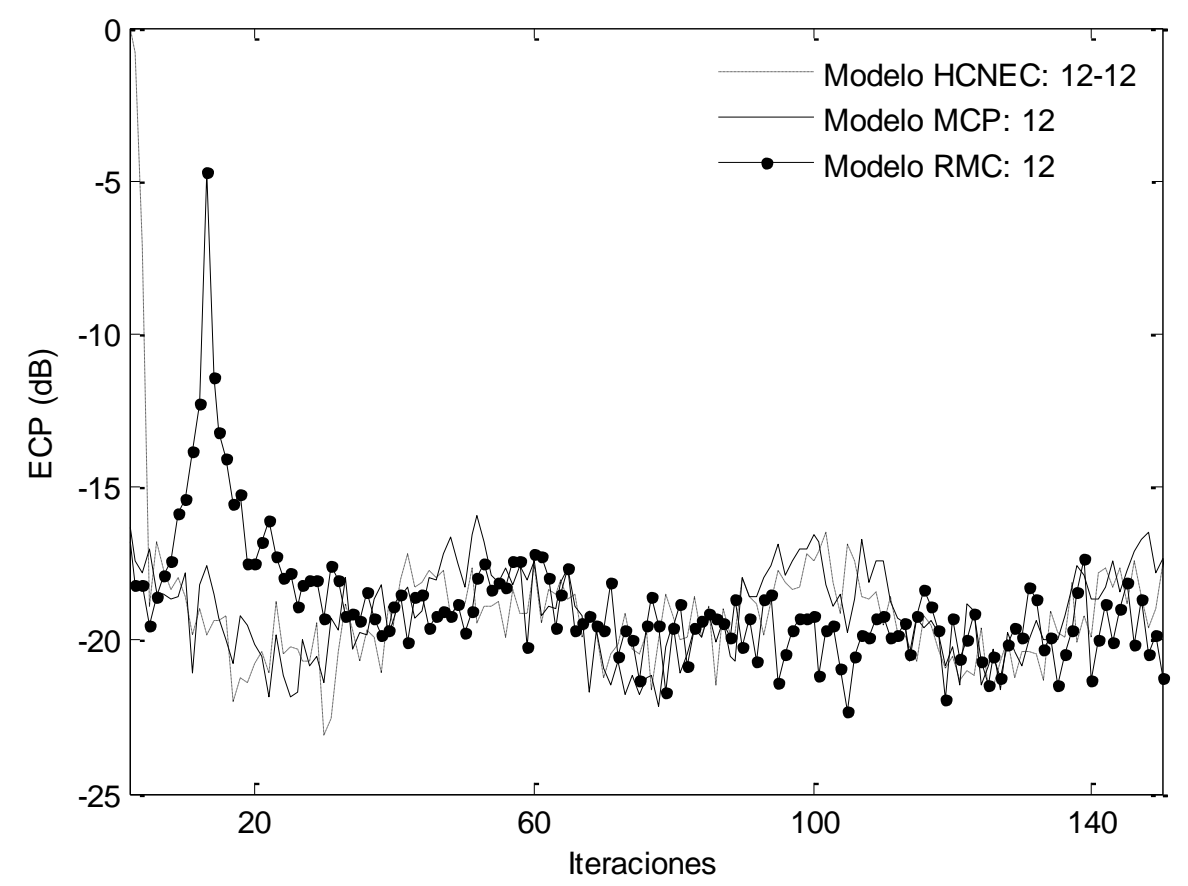

Fig. 7: Curva de aprendizaje para de los modelos HCNEC 12-12 y los algoritmos MCP y RMC de 12 antenas y/o pesos con tamaño de paso MCP- $\mu$ de 0.09 y RMC- $\lambda$ cercano a 1. Primeras 150 iteraciones. 


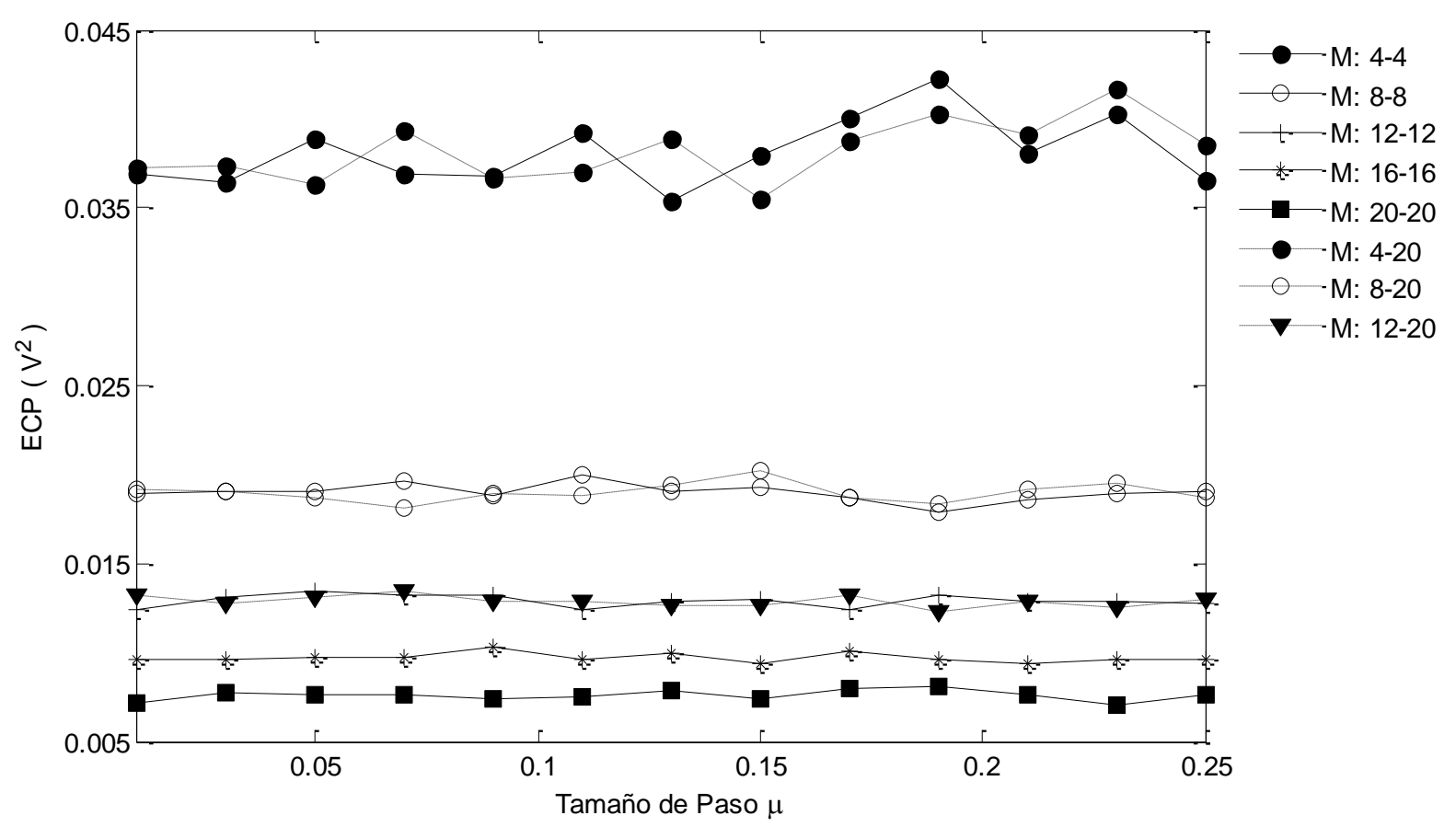

Fig. 8: ECP de acuerdo a la variación del MCP- $\mu$ en el esquema híbrido y sus variantes de acuerdo al número de antenas reales y virtuales utilizadas.

De acuerdo a los resultados mostrados, los modelos 4-4 y 4-20 presentan errores de aprendizaje ECP muy similares entre si y los más altos en su entorno de comparación. Los modelos 8-8 y 8-20 muestran características similares a los esquemas combinados anteriores, pero inferiores en cuanto al error de aprendizaje ECP. De igual manera, se observa con los modelos 12-12 y 12-20, pero con un error aún más pequeño y finalmente los modelos 20-20 y 16-16 que tienen los valores más pequeños de aprendizaje ECP, según ese orden. Una vez que el filtro ha terminado su proceso de convergencia y se ha obtenido el vector de pesos óptimos, entonces se comprueba su capacidad de recuperar la señal deseada.
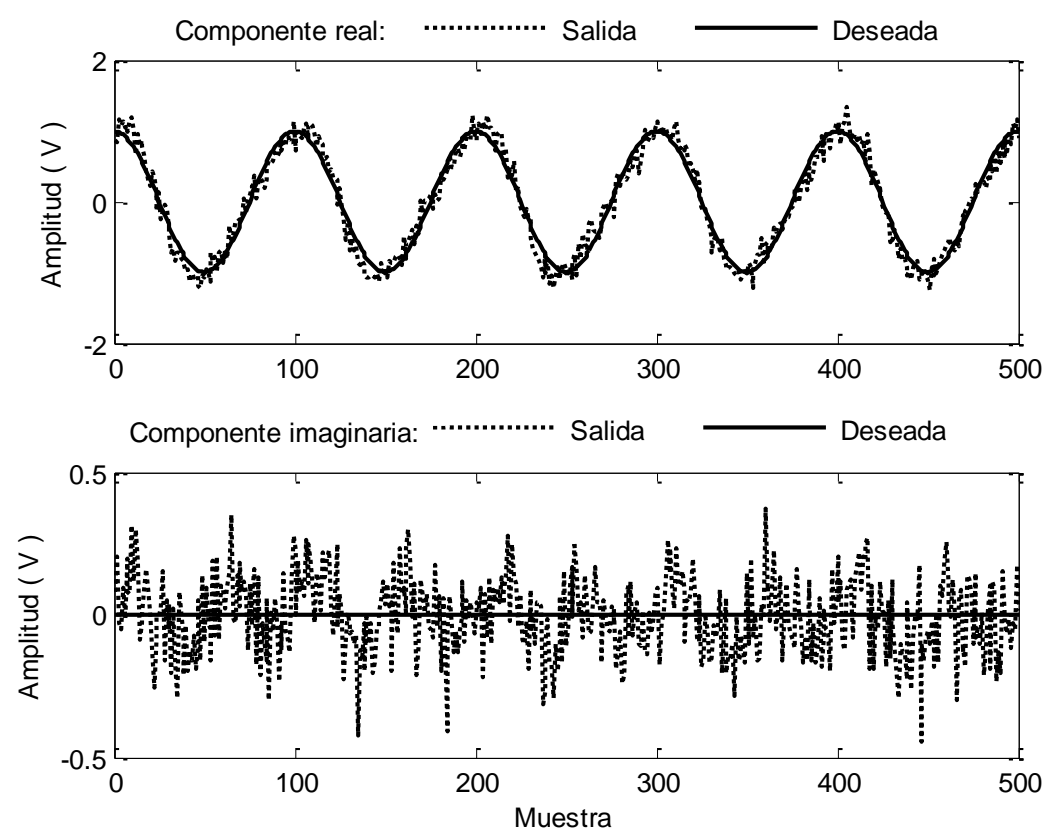

Fig. 9: Componente real e imaginaria de la señal de salida y la señal deseada en la salida del filtro para un parámetro MCP- $\mu$ de 0.09 para a un arreglo lineal híbrido 4-4. 
La figura 9 muestra las componentes real e imaginaria de la señal deseada (línea continua) y de la señal obtenida del filtro (línea entrecortada) que se obtiene a la salida del filtro híbrido 4-4 con un tamaño de paso MCP de 0.09 y la figura 10 muestra los resultados para el mismo esquema con un tamaño de paso MCP de 0.03. Por otro lado, la figura 11 muestra los resultados para un esquema HCNEC 4-20 con un tamaño de paso igual al del esquema de la figura 9.
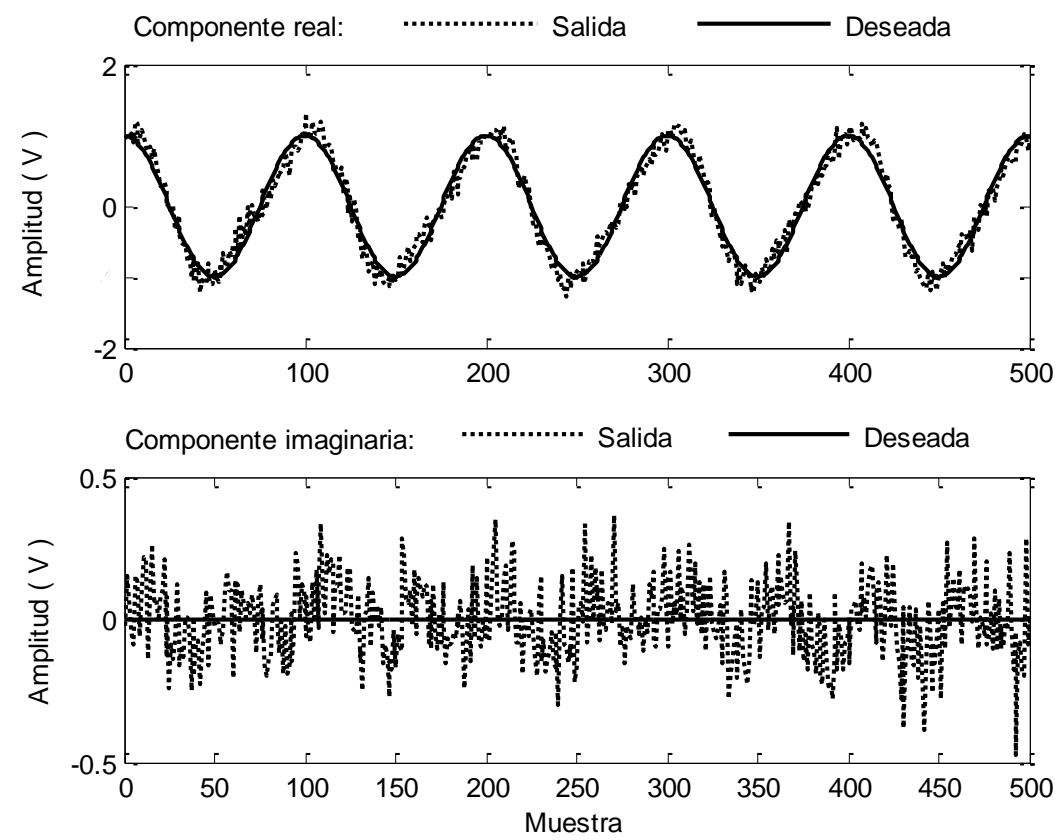

Fig. 10: Componente real e imaginaria de la señal de salida y la señal deseada en la salida del filtro para un parámetro MCP- $\mu$ de 0.03 para a un arreglo lineal híbrido 4-4.
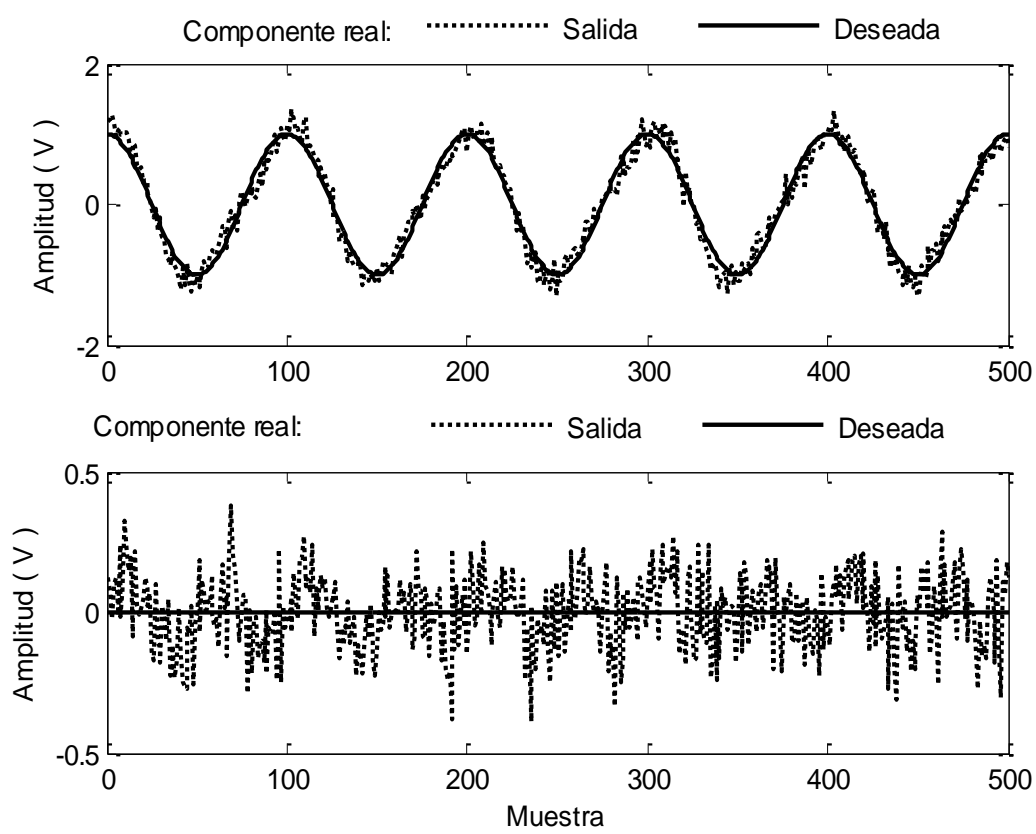

Fig. 11: Componente real e imaginaria de la señal de salida y la señal deseada del filtro HCNEC para un parámetro MCP- $\mu$ de 0.09 y un arreglo lineal híbrido 4-20.

El efecto de variar el MCP- $\mu$ en el esquema HCNEC se muestra en la figura 12 y figura 13 , en la cual se indica el error de comparación entre la señal de salida y la señal deseada denominado error de comparación (ECPA). El porcentaje de amplificación (signo positivo) o atenuación (signo negativo) se resumen en la tabla 3 y 4 tanto en la etapa de pre-filtrado como en la de pos-filtrado del esquema híbrido, respectivamente. En la figura 12 se observa que la etapa de pre-filtrado es más sensible al parámetro MCP$\mu$ conforme este aumenta, debido a que su ECPA se incrementa ya sea por una atenuación o una amplificación de la señal de salida de esta etapa. Por el contrario, la figura 13 del bloque de pos-filtrado indica que en los modelos HCNEC 4-4, 8-8, 12-12, 16-16 y 20-20, así como los combinados 4-20, 8-20 y 12- 
20, la señal de salida del filtro es más robusta con respecto al tamaño del paso debido a que su amplificación o atenuación son casi imperceptibles. El porcentaje de amplificación y atenuación se resumen en la Tabla 3 y 4 para las etapas de pre-filtrado y pos-filtrado, respectivamente.

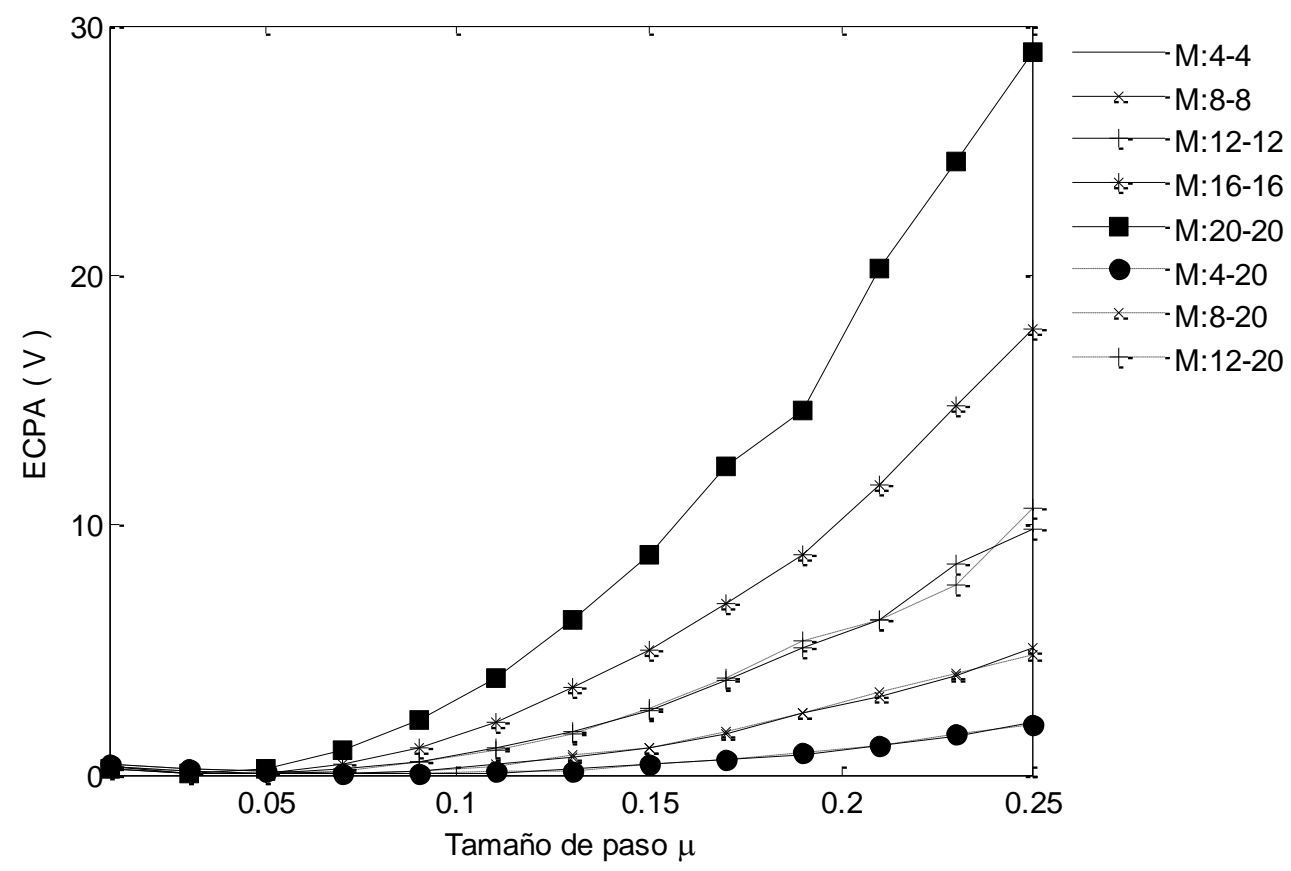

Fig. 12: Curva de ECPA del algoritmo híbrido en su etapa de pre-filtrado.

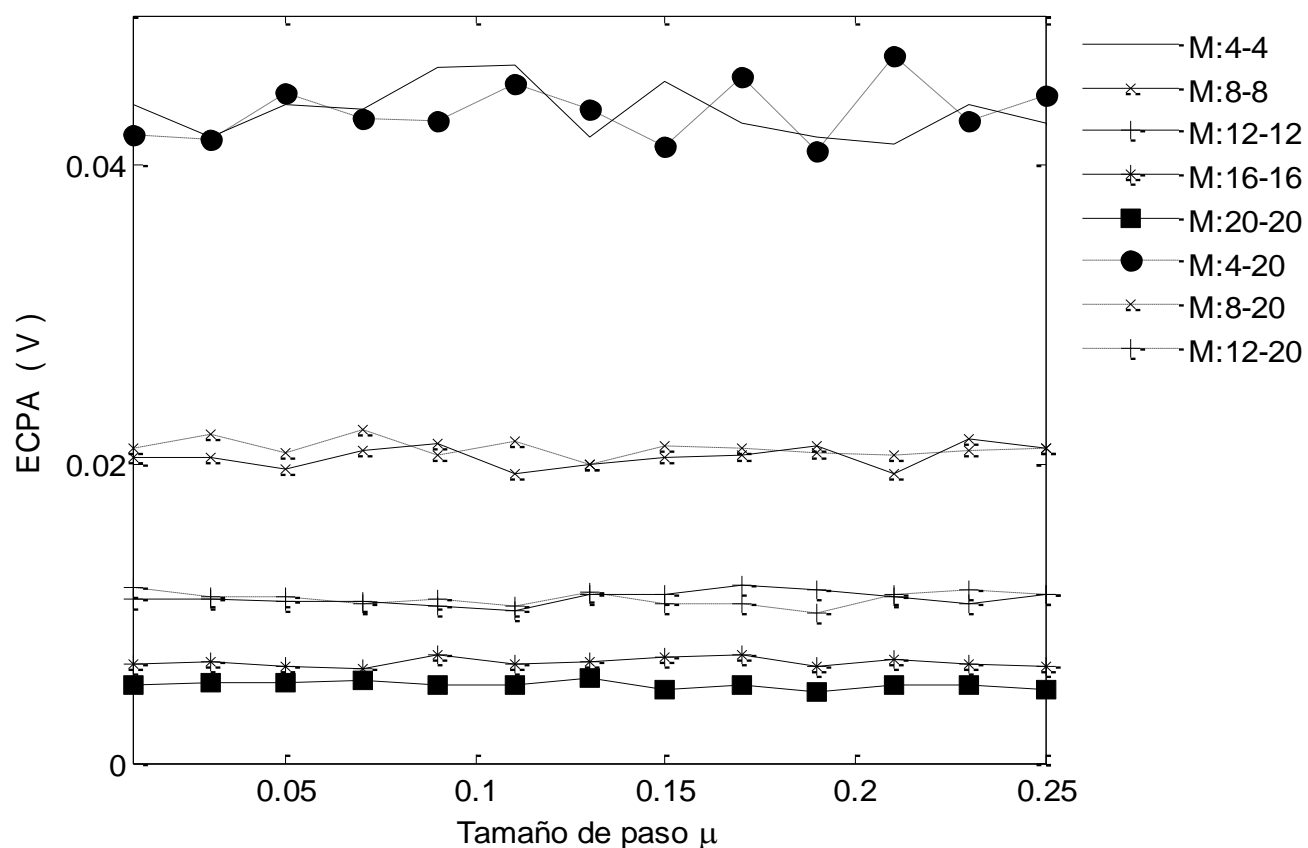

Fig. 13: Curva de ECPA del algoritmo híbrido en su etapa de pos-filtrado.

La Tabla 3 muestra un porcentaje de valores de amplificación y atenuación considerablemente altos, es decir que en base al valor del paso utilizado, la amplitud de la señal obtenida puede ser tan pequeña como 0.1 o tan grande como 10 con respecto a la amplitud de 1 que se desea. Por el contrario la Tabla 4 confirma la robustez final del algoritmo en su etapa de pos-filtrado para el esquema HCNEC ya sea con los modelos de igual número de antenas o combinados con diferentes antenas. Sus valores de amplificación y atenuación son muy pequeños, los cuales no superan en promedio valores de más allá de \pm 7 por ciento, respectivamente. El parámetro $\lambda$ del filtro RMC se ha dejado fijo en esta investigación con la intención de mostrar los efectos que se tienen en la etapa de pre-filtrado, por ser éste mucho más sensible a su parámetro que lo que realmente es el RMC con respecto al suyo. 
Tabla 3: Porcentaje de amplificación y atenuación en la etapa de pre-filtrado del algoritmo HCNEC

\begin{tabular}{|c|cccccccccccc|c|}
\hline \multirow{2}{*}{ Modelo } & \multicolumn{10}{|c|}{ Tamaño de paso del algoritmo MCP } \\
\cline { 2 - 14 } & 0.01 & 0.03 & 0.05 & 0.07 & 0.09 & 0.11 & 0.13 & 0.15 & 0.17 & 0.19 & 0.21 & 0.23 & 0.25 \\
\hline M:4-4 & -88.63 & -65.54 & -46.60 & -19.94 & 12.88 & 23.35 & 54.57 & 82.15 & 111.48 & 123.15 & 130.54 & 142.41 & 177.37 \\
\hline M:8-8 & -83.59 & -54.00 & -14.16 & 10.03 & 48.66 & 76.76 & 114.59 & 142.25 & 168.67 & 208.67 & 222.33 & 281.82 & 339.13 \\
\hline M:12-12 & -77.13 & -35.58 & 7.53 & 51.32 & 90.45 & 132.39 & 179.14 & 215.23 & 268.56 & 305.88 & 348.71 & 408.49 & 419.23 \\
\hline M:16-16 & -71.94 & -16.97 & 34.70 & 88.24 & 143.21 & 200.85 & 266.43 & 320.36 & 380.84 & 445.50 & 452.32 & 533.30 & 613.86 \\
\hline M:20-20 & -64.71 & 5.30 & 71.29 & 136.87 & 197.80 & 271.15 & 360.22 & 426.81 & 493.77 & 525.42 & 619.92 & 709.69 & 764.57 \\
\hline M:4-20 & -88.88 & -63.53 & -42.89 & -20.26 & 3.05 & 32.52 & 44.48 & 81.96 & 90.98 & 111.27 & 124.16 & 158.00 & 154.24 \\
\hline M:8-20 & -83.56 & -52.93 & -18.74 & 18.23 & 45.21 & 78.85 & 110.79 & 139.11 & 162.97 & 192.74 & 262.15 & 305.30 & 293.46 \\
\hline M:12-20 & -78.79 & -40.39 & 8.71 & 47.25 & 99.53 & 131.06 & 178.92 & 213.29 & 268.28 & 327.10 & 368.57 & 403.77 & 468.44 \\
\hline
\end{tabular}

Tabla 4: Porcentaje de amplificación y atenuación en la etapa de pos-filtrado del algoritmo HCNEC

\begin{tabular}{|c|cccccccccccccc|}
\hline \multirow{2}{*}{ Modelo } & \multicolumn{10}{|c|}{ Tamaño de paso del algoritmo MCP } \\
\cline { 2 - 14 } & 0.01 & 0.03 & 0.05 & 0.07 & 0.09 & 0.11 & 0.13 & 0.15 & 0.17 & 0.19 & 0.21 & 0.23 & 0.25 \\
\hline M:4-4 & $-6,07$ & $-7,38$ & $-2,57$ & $-2,08$ & 1,19 & 10,86 & $-3,50$ & 2,02 & 6,43 & 6,03 & 0,69 & $-2,75$ & $-2,76$ \\
\hline M:8-8 & $-3,05$ & $-13,55$ & $-0,09$ & $-0,55$ & 3,74 & 0,63 & $-1,88$ & 0,89 & $-7,67$ & 3,75 & 1,87 & $-2,96$ & $-6,02$ \\
\hline M:12-12 & $-1,39$ & $-4,11$ & $-0,02$ & 3,61 & 2,71 & $-4,18$ & 1,72 & $-2,67$ & $-0,83$ & 0,72 & $-3,41$ & $-0,02$ & $-0,52$ \\
\hline M:16-16 & -1.78 & 0.23 & -1.07 & -2.10 & -0.17 & -0.10 & 1.70 & 1.45 & 3.24 & 5.01 & -5.03 & -0.62 & 3.96 \\
\hline M:20-20 & -0.53 & 1.10 & 0.43 & -1.06 & -2.78 & -1.66 & 2.15 & 1.64 & 0.10 & -1.62 & -1.00 & 1.75 & 0.96 \\
\hline M:4-20 & -4.15 & 6.31 & -0.43 & 2.99 & 1.04 & 5.03 & 1.66 & 5.85 & -1.64 & -0.21 & -3.30 & -0.86 & -8.11 \\
\hline M:8-20 & -0.55 & -3.30 & 0.15 & 1.34 & -0.99 & 0.04 & -2.67 & -2.09 & -6.63 & -7.69 & 2.21 & 5.61 & -3.00 \\
\hline M:12-20 & -1.66 & -7.30 & -0.46 & -1.57 & 1.05 & -2.30 & -0.57 & -3.95 & -1.44 & 0.62 & 3.97 & 2.42 & 1.90 \\
\hline
\end{tabular}

Debido a que no es adecuado comparar el costo computacional del algoritmo HCNEC con los algoritmos MCP y RMC, ya que estos constituyen el esquema híbrido y evidentemente tienen un costo computacional inferior por sí mismos, se ha recurrido y sólo para efectos de comparación a otros algoritmos en cascada con características similares al propuesto en este artículo. De acuerdo a la figura 14 y 15 el algoritmo propuesto en todos los casos es inferior a los modelos: Hibrido en Cascada Independiente $(\mathrm{HCl})$ e Hibrido en Cascada No Independiente ( $\mathrm{HCNI}$ ) (Orozco et al, 2013) y al propuesto por Srar y Chung (2009) denominado RMC-MCP.

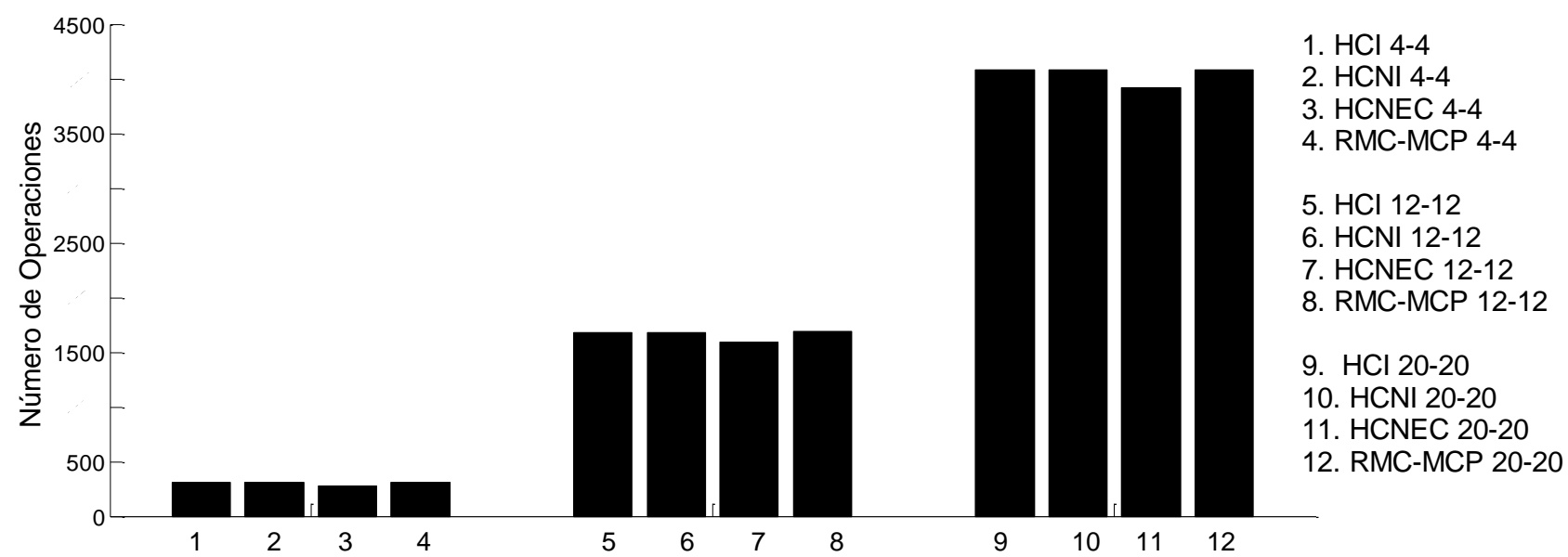

Fig. 14: Gráfica comparativa del costo computacional utilizado por iteración del algoritmo HCNEC frente a otros algoritmos en cascada que utilizan algoritmos MCP y RMC. Arreglos de antenas con igual número de antenas reales y virtuales. 


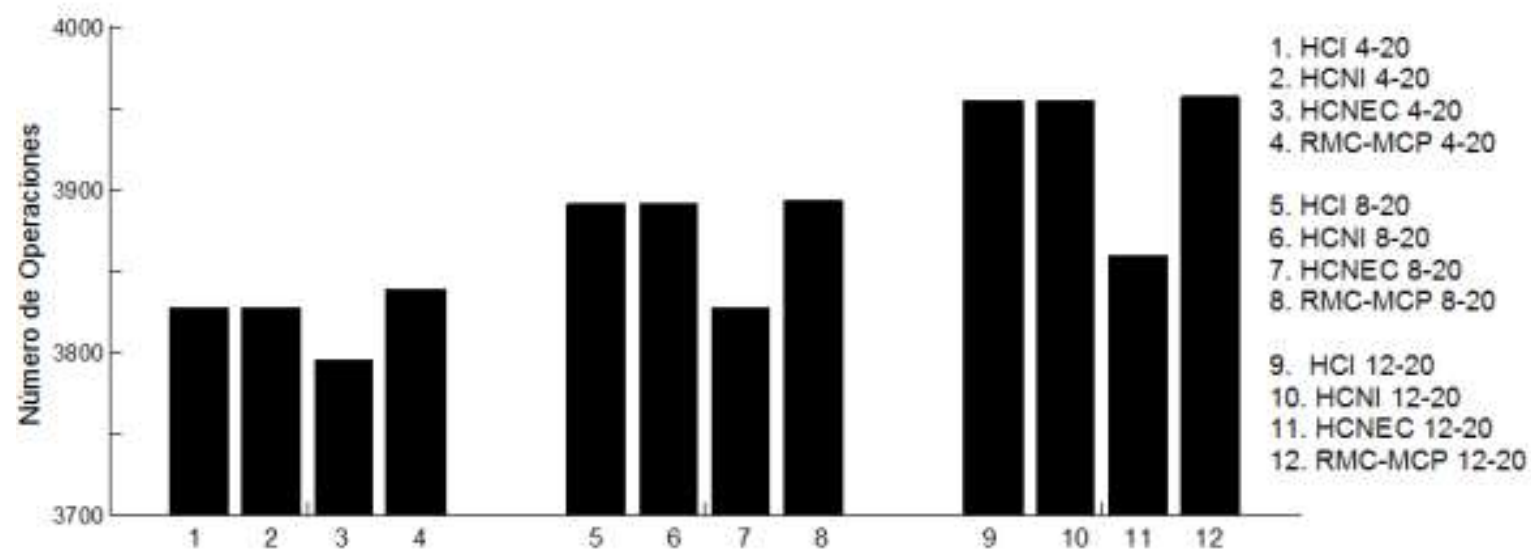

Fig. 15: Gráfica comparativa del costo computacional utilizado por iteración del algoritmo HCNEC frente a otros algoritmos en cascada que utilizan algoritmos MCP y RMC. Arreglos de antenas con diferente número de antenas reales y virtuales.

Las figuras 16 y 17 muestran que los esquemas HCNIE 12-12 y 16-16, tomados como ejemplo para mostrar los resultados, fueron capaces de generar el lóbulo de radiación principal hacia la dirección de la señal que se pretendía obtener, cuyo ángulo de incidencia corresponde a los 60 grados. En general, las diferentes combinaciones de antenas utilizadas en este análisis mostraron lóbulos de radiación similares.

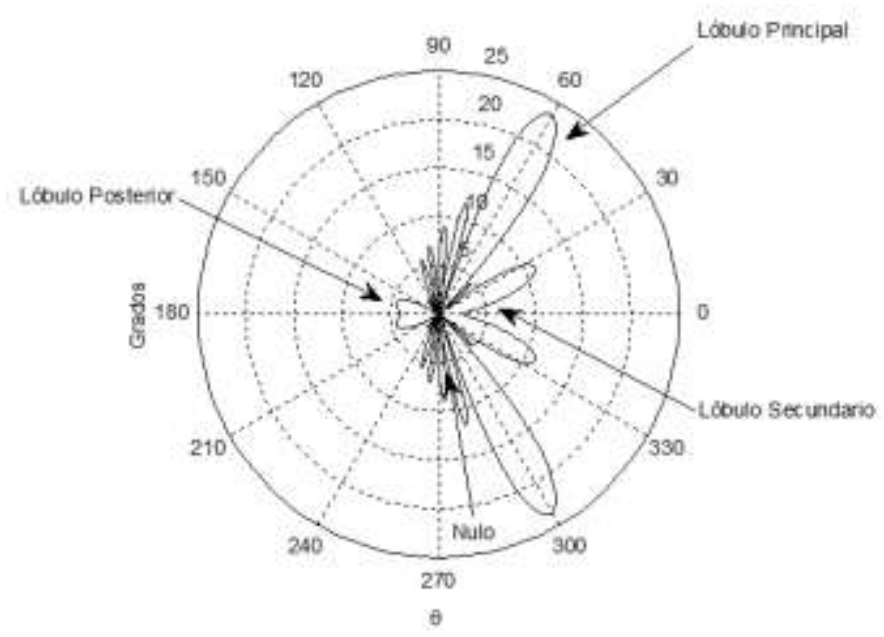

Fig. 16: Lóbulos de radiación generados por un HCNEC 12-12. Parámetro MCP- $\mu$ de 0.09.

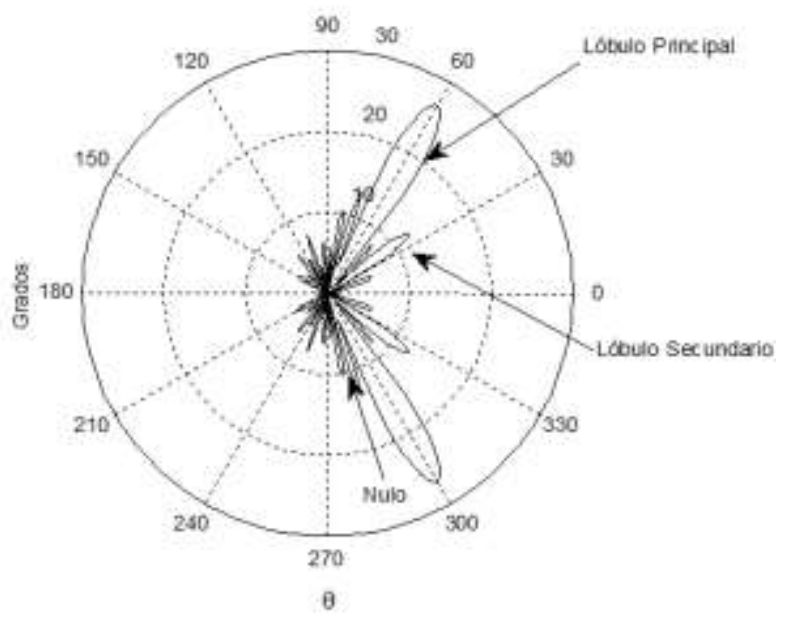

Fig. 17: Lóbulos de radiación generados por un HCNEC 16-16. Parámetro MCP- $\mu$ de 0.09. 


\section{CONCLUSIONES}

En este artículo se propone un esquema híbrido denominado HCNEC formado en base a los algoritmos simples MCP y RMC con una señal de error común. El análisis matemático involucrado en su formulación demuestra que las ecuaciones del vector de pesos concerniente a cada bloque adaptativo así como el vector combinado de pesos convergen a la solución de Wiener. De igual manera lo hacen las ecuaciones de error cuadrado promedio y error mínimo cuadrado.

Los resultados experimentales con respecto al número de iteraciones requeridas para alcanzar el EMCP muestran una rapidez de convergencia similar a la de un esquema simple MCP y mucho mejor que la de un algoritmo RMC. Esto implica que aunque el algoritmo híbrido este conformado por dos filtros en serie, no necesariamente implica un decremento en su rapidez de convergencia. De igual manera se observa que su capacidad de filtrado depende del número de antenas involucradas en el arreglo, tanto en el filtro de entrada como en el bloque de salida cuyas antenas se simulan usando el VAL. Generalmente el aumento de las antenas implica una mejor resolución en la señal de salida y por lo tanto una disminución en el error de comparación entre la señal obtenida usando el mismo tamaño de paso del pre-filtro MCP. Por otro lado, la variación del parámetro $\mathrm{MCP}-\mu$ ofrece la posibilidad de obtener una característica importante de amplificación y atenuación del filtro en su etapa de pre-filtrado, ya que la señal de salida obtenida en este bloque de procesamiento sigue manteniendo la misma frecuencia y fase que la señal de información deseada y tan solo modifica su amplitud. Por el contrario, en la etapa de pos-filtrado la variación del parámetro MCP- $\mu$ no tiene un efecto significante de amplificación y atenuación en la amplitud de la señal de salida final, generando así una señal de características similares a la deseada. Esto implica que el sistema como tal es capaz de minimizar la contaminación por RBGA y la señal de interferencia involucrada.

El cálculo del costo computacional con respecto a otros esquemas híbridos de características similares muestra que el filtro en sí es un modelo menos complejo por iteración. Finalmente, el filtro es capaz de generar el lóbulo de radiación principal hacia la dirección de la señal de información que se desea obtener, produciendo lóbulos laterales nulos en otras direcciones. No obstante es importante recalcar, que dicho lóbulo de radiación puede variar en forma y área de cobertura dependiendo una vez más del número de antenas utilizadas en el arreglo lineal y generar una señal no tan pura libre de interferencia y RBGA.

\section{AGRADECIMIENTOS}

Los autores agradecen al Consejo Nacional de Ciencia y Tecnología (CONACyT) y al Instituto Politécnico Nacional por el apoyo otorgado para la realización de este trabajo.

\section{NOTACION}

$\begin{array}{ll}\text { Abreviatura } & \text { Significado } \\ \text { AAD } & \text { Ángulo de Arribo Deseado } \\ \text { AES } & \text { Alisado Exponencial Simple } \\ \text { CMCP } & \text { Cascada de Mínimos Cuadrados Promediados } \\ \text { DMOF } & \text { División Multiplexada Ortogonal de Frecuencia } \\ \text { ECP } & \text { Error al Cuadrado Promediado } \\ \text { ECPA } & \text { Error de Comparación } \\ \text { EMCP } & \text { Error Mínimo Cuadrático Promediado } \\ \text { HCI } & \text { Hibrido en Cascada Independiente } \\ \text { HCNEC } & \text { Híbrido en Cascada No independiente con Error Común } \\ \text { HCNI } & \text { Hibrido en Cascada No Independiente } \\ \text { IMM } & \text { Inversión de la Matriz de Muestras } \\ \text { MCP } & \text { Mínimos Cuadrados Promediados } \\ \text { MCPC } & \text { Mínimos Cuadrados Promediados Coherente } \\ \text { MCP -CN } & \text { Mínimos Cuadrados Promediados Coherente Normalizado } \\ \text { MPNC } & \text { Mínimos Promediados de Norma Combinada } \\ \text { RBGA } & \text { Ruido Blanco Gaussiano Aditivo } \\ \text { RMC } & \text { Recursivo de Mínimos Cuadrados }\end{array}$




$\begin{array}{ll}\text { RSDMV } & \text { Respuesta Sin Distorsión de Mínima Varianza } \\ \text { RSR } & \text { Relación Señal a Ruido } \\ \text { RSRI } & \text { Relación Señal a Ruido más Interferencia } \\ \text { TFD } & \text { Transformada de Fourier Discreta } \\ \text { TRF } & \text { Transformada Rápida de Fourier } \\ \text { VAL } & \text { Vector de Arreglo Lineal }\end{array}$

\section{REFERENCIAS}

Acevedo, M.A. y otros tres autores, A Novel Method of Beamforming to Improve the Space Diversity, Polibits: (46), 93-98 (2012).

Diniz, P., Adaptive Filtering algorithms and Practical Implementation, $4^{\text {ta }}$ edición, 36-41, Springer, New York, USA (2013).

Fernández, S.G. y otros tres autores, Optimización de la Estimación de DOA en Sistemas de Antenas Inteligentes usando criterios de Redes Neuronales, RIELAC: 34(1), 70-86 (2013).

Haykin, S., Communication Systems, $4^{\text {ta }}$ edición, 3, Jhon Wiley \& Sons, New York, USA (2001).

Kraus, J. y R. Marhefka, Antennas For All Applications., 3ra edición, 109-120, McGraw-Hill Higher Education., New York, USA (2002).

Liang, Y. y F. Chin, Coherent LMS Algorithm, IEEE Communications Letters, 4(3), 92-94 (2000).

Orozco-Tupacyupanqui, W., M. Nakano-Miyatake y H. Pérez-Meana., Adaptive Cascade Hybrid Configurations for Linear Array Beamforming, NAUN International Journal of Communications: 7(3), 55-66 (2013).

Rahmani, M., M.H. Bastani y S. Shahraini, Two layers beamforming robust against direction-of-arrival mismatch, IET Signal Processing: 8(1), 49-58 (2014).

Sayed, A.H., Fundamentals of Adaptive Filtering, $1^{\text {a }}$ edición, 245-248, John Wiley \& Sons, Inc., New Jersey, USA (2003).

Shi., L. y otros cinco autores, An Improved Real-Coded Genetic Algorithm for the Beam Forming of Spaceborne SAR, IEEE Transaction on Antennas and Propagation: 60(6), 3034-3040 (2012).

Shubair, R.M., Robust adaptive Beamforming using LMS Algorithm with SMI Initialization, IEEE Antennas and Propagation Society International Symposium, 2-5, Washington, DC.,USA, 3 al 8 de Julio (2005).

Shubair, R.M. y S.A. Jimma, Improved Adaptive Beamforming Using the Least Mean Mixed-Norm Algorithm, $9^{\text {th }}$ International Symposium on Signal Processing and Its Applications, 1-4, Sharjah, United Arab Emirates, 12 al 15 de Febrero (2007).

Srar, J. y K. Chung, Adaptive RLMS Algorithm for Antenna array Beamforming, IEEE $10^{\text {th }}$ Conference TENCON, 1-6, Singapore, Malaysia, 23 al 26 de Noviembre (2009).

Tuan, L. y otros tres autores, A New RLs-based adaptive Beamforming algorithm for Smart Antennas Applied to an OFDM System, $3^{\text {rd }}$ International Conference on Microwave and Millimeter Wave Technology, 672 - 675, Beijing, China, 17 al 19 de Agosto (2002).

Watanabe, K. y R. Kohno, Hybrid Adaptive Algorithm Based on Temporal Update and Spatial Spectrum Estimation for Adaptive Array Antenna, IEEE Vehicular Technology Conference, 175-180, Boston, USA, 24 al 28 de Septiembre (2000).

Widrow, B. y S.D Stearns, Adaptive Signal Processing, $1^{\text {a }}$ edición, 99-103, Prentice-Hall Signal Processing, New Jersey, USA (1985).

Yang, X. y otros tres autores, Applying Auxiliary Array to Suppress Mainlobe Interference for Ground-Based Radar, IEEE Antennas and Wireless Propagation Letters: (12), 433-436 (2013). 
